OPEN ACCESS

Edited by: Chunhong Yan,

Augusta University, United States

Reviewed by:

Lian Xiang Luo,

Guangdong Medical University, China

Jing Jin,

Chinese Academy of Medical Sciences and Peking Union Medical

College, China

*Correspondence: Zuojia Liu zjliu@ciac.ac.cn Jin Wang

jin.wang.1@stonybrook.edu Erkang Wang ekwang@ciac.ac.cn

Specialty section: This article was submitted to Pharmacology of Anti-Cancer Drugs,

a section of the journal

Frontiers in Pharmacology

Received: 19 October 2021 Accepted: 22 November 2021

Published: 05 January 2022

Citation:

Zhang J, Liu Z, Zhao W, Yin X, Zheng X, Liu C, Wang J and Wang E (2022) Discovery of Small Molecule NSC290956 as a Therapeutic Agent for KRas Mutant Non-Small-Cell

Lung Cancer.

Front. Pharmacol. 12:797821. doi: 10.3389/fphar.2021.797821

\section{Discovery of Small Molecule NSC290956 as a Therapeutic Agent for KRas Mutant Non-Small-Cell Lung Cancer}

\author{
Jiaxin Zhang ${ }^{1,2}$, Zuojia Liu ${ }^{1 *}$, Wenjing Zhao ${ }^{1}$, Xunzhe Yin ${ }^{1}$, Xiliang Zheng ${ }^{1}$, Chuanbo Liu ${ }^{1}$, \\ Jin Wang ${ }^{3 *}$ and Erkang Wang ${ }^{1,2 *}$
}

${ }^{1}$ State Key Laboratory of Electroanalytical Chemistry, Changchun Institute of Applied Chemistry, Chinese Academy of Sciences, Changchun, China, ${ }^{2}$ Department of Chemistry, University of Science and Technology of China, Hefei, China, ${ }^{3}$ Department of Chemistry and Physics, State University of New York, Stony Brook, NY, United States

HRas-GTP has a transient intermediate state with a "non-signaling open conformation" in GTP hydrolysis and nucleotide exchange. Due to the same hydrolysis process and the structural homology, it can be speculated that the active KRas adopts the same characteristics with the "open conformation." This implies that agents locking this "open conformation" may theoretically block KRas-dependent signaling. Applying our specificity-affinity drug screening approach, NSC290956 was chosen by high affinity and specificity interaction with the "open conformation" structure HRasG60A-GppNp. In mutant KRas-driven non-small-cell lung cancer (NSCLC) model system, NSC290956 effectively suppresses the KRas-GTP state and gives pharmacological KRas inhibition with concomitant blockages of both the MAPK-ERK and AKT-mTOR pathways. The dual inhibitory effects lead to the metabolic phenotype switching from glycolysis to mitochondrial metabolism, which promotes the cancer cell death. In the xenograft model, NSC290956 significantly reduces H358 tumor growth in nude mice by mechanisms similar to those observed in the cells. Our work indicates that NSC290956 can be a promising agent for the mutant KRas-driven NSCLC therapy.

Keywords: specificity-affinity screening, cancer metabolism, drug discovery, non-small-cell lung cancer, open conformation of Ras

\section{INTRODUCTION}

Ras proteins function in many signaling pathways as a binary molecular switch cycling between an active guanosine triphosphate (GTP)-bound (RasT) and an inactive guanosine diphosphate (GDP)bound (RasD) state (Pylayeva-Gupta et al., 2011; Hobbs et al., 2016). In tumor, Ras is stuck in a permanently active RasT state (Burns et al., 2014). At this form, RasT continuously activates diverse down-stream effectors. Subsequently, the activated state is terminated by converting RasT to RasD state. Proper regulation of Ras cycling is essential for cellular functions; thus, the dysregulation is a hallmark of tumor (Hanahan and Weinberg, 2011).

GTP hydrolysis is in equilibrium with three distinct but equally populated conformations, one of which is the "non-signaling open conformation" (Ford et al., 2005). Nassar et al. revealed that HRasG60A-GppNp complex was found to adopt an "open conformation" at the switch 1 region and to abolish the biological activity of HRas (Hwang et al., 1996; Ford et al., 2005). Initially these results 


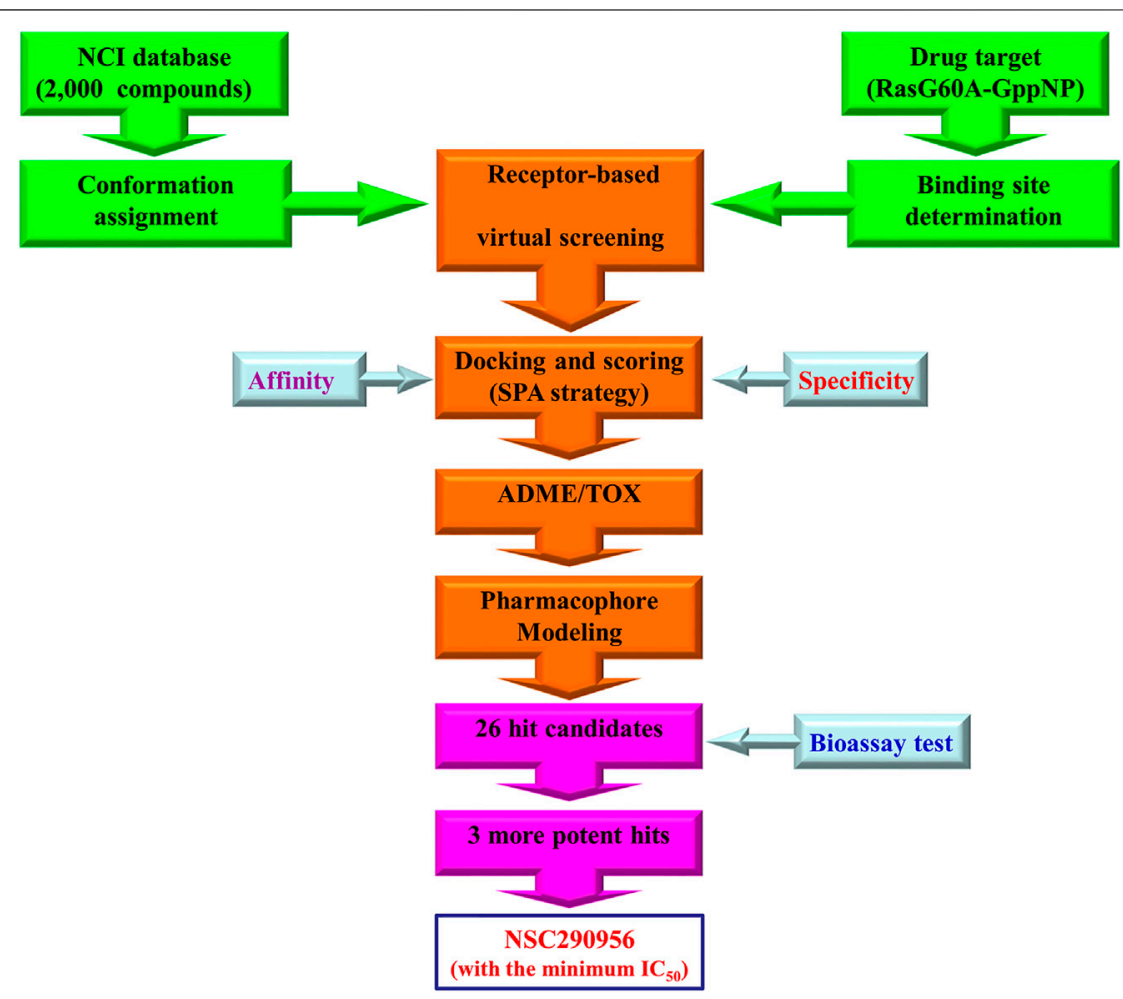

FIGURE 1 | Workflow of specificity-affinity virtual screening strategy. Out of this screening, 26 small-molecule compounds were selected for further bioactivity testing with three hits discovered. Thereafter, NSC290956 was picked out due to its minimum $\mathrm{IC}_{50}$ value.

were obtained by using HRas proteins, but the states also occur in KRas (Parker et al., 1993). In cancer cells the mutant KRas accumulates in an elevated GTP-bound proportion and thus leads to a constantly activated form. Once at the KRasT state, this reaction of GTP hydrolysis and nucleotide exchange occurs immediately. Insights into KRas dynamic from experimental data showed that specific mutations in the switches (D33E in switch 1 or A59G in switch 2) (Lu et al., 2018), replacing Y32 in switch 1 with other amino acids (Spoerner et al., 2010), and phosphorylating tyrosine residues Y32 in switch 1 and Y64 in switch 2 induced the equilibrium towards an "open conformation" (Kano et al., 2019). Recent crystal structures combined with molecular dynamic simulations indicated extremely open switch 1 conformations of KRas (Pantsar, 2020). This implies that the "open conformation" may be a convergent point for survival signaling in KRas-driven cancer.

Given frequent addiction to oncogenic KRas, targeting the transition state structure would be therapeutically beneficial. (1) Targeting a transition state structure by drug design was known as an effective approach for inhibiting an enzymatic reaction. (2) Global genomic analysis revealed that drugs targeting key nodal points could be preferable for therapies. In principle, a drug directed at targeting this intermediate transition conformation might act as a "gatekeeper" that keeps KRas in a conformation incompatible with downstream effectors binding through decreasing the proportion of KRasT state (Sun et al., 2012). Along this line, such a drug ultimately has a pharmacological inhibition of KRas oncoproteins, thereby interrupting KRas-dependent survival in the cell.
In this study, we take advantage of the finding to develop small molecule inhibitors that stabilize the "open conformation" of activated KRas in tumor cell, thus preventing KRas from converting to its signaling one. Given the nearly $25 \%$ frequency of KRas mutations in non-small-cell lung cancer (NSCLC), we set out to utilize NSCLC as a model system to identify this novel antitumor strategy for targeting the intermediate conformation structure. We use a multidisciplinary approach based on the synergy of computational, biophysical, and biological methods to identify the "open conformation" as a potential target. Applying a specificity-affinity virtual screening workflow (Figure 1), we discover a small molecule NSC290956. Combining data from ensemble docking in silico, binding interactions in vitro and in vivo, and experiments in intact cells and mouse models, we show that NSC290956 specifically binds to the "open conformation" structure, effectively attenuates KRas functions, and significantly reduces NSCLC cell survival and xenograft tumor growth in mice. This work provides a functional insight into the "open conformation" and validates a promising therapeutic strategy for KRas-dependent cancer.

\section{RESULTS}

\section{Virtual Screening and Hit Validation}

Comparing the conformation difference between GppNp-bound HRasG60A (PDB: 1XCM) and HRas (PDB: 2RGE), an 

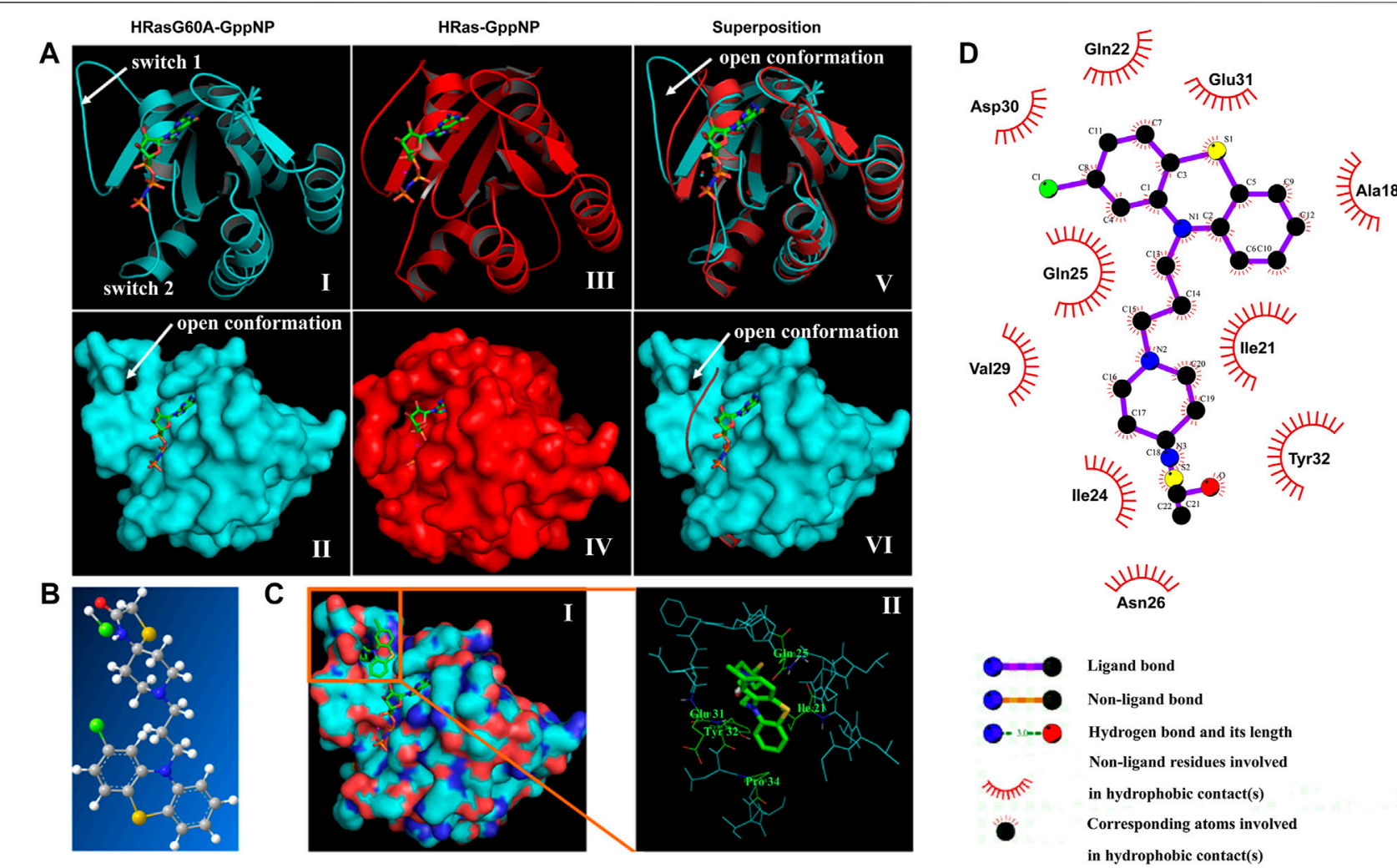

FIGURE 2 | In silico virtual screening identifies NSC290956 as a hit compound. (A) Structure models of HRasG60A-GppNp (PDB ID: 1XCM), HRas-GppNp (PDB ID: 2RGE), and superposition of the two structures as a ribbon (top) and surface (bottom) representations. The switch regions (switch 1 \& 2 ) are indicated. GppNp is shown as a stick model. Mg $^{2+}$ is shown as a cyan sphere in HRasG60A-GppNp or red sphere in HRas-GppNp, respectively. (B) Chemical structure of NSC290956 (C, grey; O, red; N, blue; H, white; S, yellow; and Cl, green). (C) Surface representation shows NSC290956 binding to the cavity on HRasG60A-GppNp. GppNp and NSC290956 are shown as stick models (i). Residues involving intermolecular interactions and NSC290956 (green) are shown on the backbone structure of binding cavity of HRasG60A-GppNp (ii). (D) Schematic diagram shows putative interaction between NSC290956 and HRasG60A-GppNp. Compound is shown in purple stick (C, black; O, red; N, blue; S, yellow; and $\mathrm{Cl}$, green). Residues involving hydrophobic interactions are shown as starbursts.

unexpected characteristic of HRasG60A-GppNp structure is the remodeling of the dynamic at the switch 1 region to a conformation that completely pulls it away from nucleotide into solvent (Figures 2Ai,iii). And the dynamic at switch 2 region is restructured and moves away from nucleotide (Ford et al., 2005). Superposition shows that mutating Gly60 to Ala leads to a dramatic opening of switch 1 (images $\mathrm{v}$ and vi). This means that an "open conformation" is formed on the surface of HRasG60A-GppNp. Structural analysis further shows that the "open conformation" is remarkably similar to the nucleotide free HRas (NF-HRas) as found in the HRas/Sos complex (BoriackSjodin et al., 1998), which constitutes an intermediate for nucleotide exchange (Lenzen et al., 1998). Accordingly, HRasG60A-GppNp has the same characteristic with the transient intermediate for nucleotide exchange right after GTP binding to NF-HRas but before the final GTP-bound conformation was reached (Ford et al., 2005). Consequently, the "open conformation" possesses a dominant negative characteristic, losing its ability to activate downstream effectors in the cells (Sung et al., 1996; Ford et al., 2005).

The surface cavity as an "open conformation" (images ii and iv) to be frozen appears as a target suitable for drug binding. We used a two-dimension screening strategy to dock compounds to this binding cavity individually. This process led to the selection of 26 compounds by scoring the binding energies and calculating the intrinsic specificity ratios (ISR) (Supplementary Tables S1, S2). After testing these candidates, we focused on the most promising compound NSC290956 (Figure 2B; Supplementary Figure S1A). In in silico docking model, NSC290956 was trapped in the binding cavity between the switch 1 region and the relevant residues (Figure $2 \mathrm{C}$ ).

The predicted interaction between NSC290956 and HRasG60A-GppNp was generated by the computational model (Figure 2D). This hydrophobic cavity is occupied by the aromatic phenothiazine group of NSC290956 with chloro substitute providing tight van der Waals contacts. The corresponding atoms contained in the phenothiazine group lead to hydrophobic interactions with residues Ala18, Gln22, Asp30, and Glu31. The diazaspiro decane group of the warhead of NSC290956 is inserted into the cavity pocket, leading to hydrophobic interactions with residues Ile21, Ile24, and Tyr32. Accordingly, an extensive hydrogen bonding network is formed between NSC290956 and the corresponding residues in the switch 1 region. 


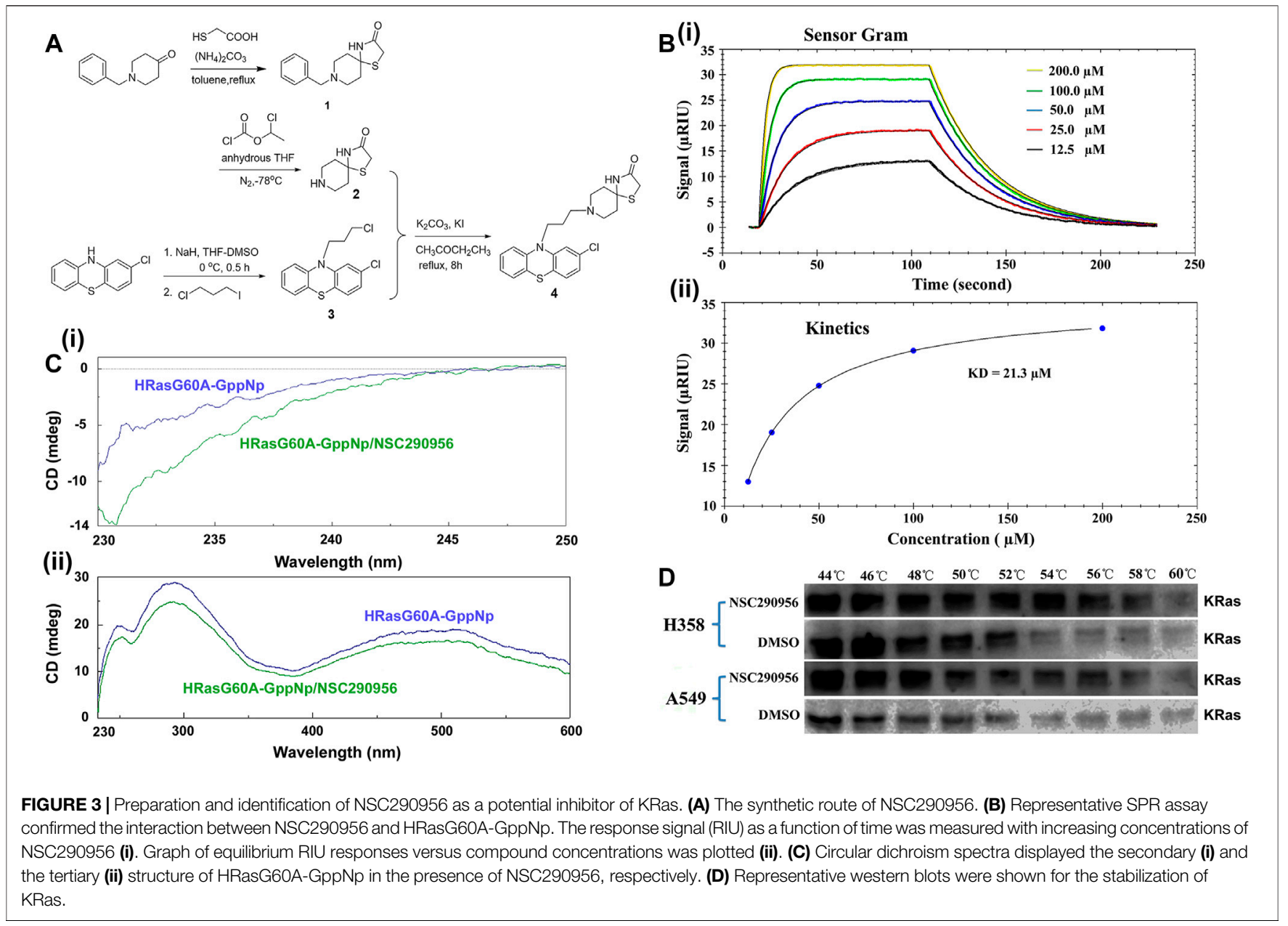

\section{Preparation and Identification of NSC290956 as a Potential Inhibitor of KRas}

The hit candidate NSC290956, chemical name 1-Thia-4,8diazaspiro[4.5]decan-3- one,8-[3-(2-chloro-10H-phenothiazin10 -yl)propyl], was prepared by following the synthetic route illustrated in the scheme in Figure $\mathbf{3 A}$ and confirmed by ESIMS spectra (Supplementary Figure S1B). The detailed synthetic procedures and characterizations of intermediate compounds, including the original NMR and ESI-MS spectra, are described in the Supplementary Material. Subsequently, the interaction between NSC290956 and the HRasG60A-GppNp complex was experimentally identified by the surface plasmon resonance (SPR) assay. The binding curves of association/dissociation and the steady-state analysis disclosed a high affinity with $\mathrm{KD}$ value of $21.3 \mu \mathrm{M}$ (Figure 3B). In contrast, HRas-GppNp did not appear to interact with NSC290956 in the identical conditions (Supplementary Figure S2). This observation clearly demonstrates that the specific binding pocket only exists in HRasG60A-GppNp. In addition, circular dichroism spectroscopy uncovered that the secondary and the tertiary structure of HRasG60A-GppNp was respectively disrupted with the addition of NSC290956 (Figure 3C), which may be a strong identification for the specific interaction.
Considering the nearly $25 \%$ frequency of KRas mutations in non-small-cell lung cancer (NSCLC), we set out to use diverse KRas-expressing NSCLC cell models to identify this novel antitumor strategy. In mutant KRas-driven cancer cells, the "open conformation" dominantly results from the activated KRas due to the "open conformation" accounting for more partition in mutated KRas than in wild-type HRas and NRas (Ford et al., 2005; Janes et al., 2018). Based on the target engagement principle (Martinez Molina et al., 2013), we performed the cellular thermal shift assay (CETSA) to verify the cellular uptake and target engagement of NSC290956 in KRas-addicted H358 (KRasG12C) and A549 (KRasG12S) cell lines. KRas alone (DMSO as control) denatured around $52-54^{\circ} \mathrm{C}$ within cells. The addition of NSC290956 $(25 \mu \mathrm{M})$ stabilized KRas by $4-6^{\circ} \mathrm{C}$ (Figure 3D). The observations indicate that NSC290956 directly binds to and thermally stabilizes KRas to a certain extent with increasing the denaturation temperature. Combining the specific in vitro interaction between NSC290956 and the "open conformation" structure, the direct in vivo interaction supports the existence of the "open conformation" structure in the activated KRas cells as the cellular target of NSC290956 in the subsequent cell-based assays. 


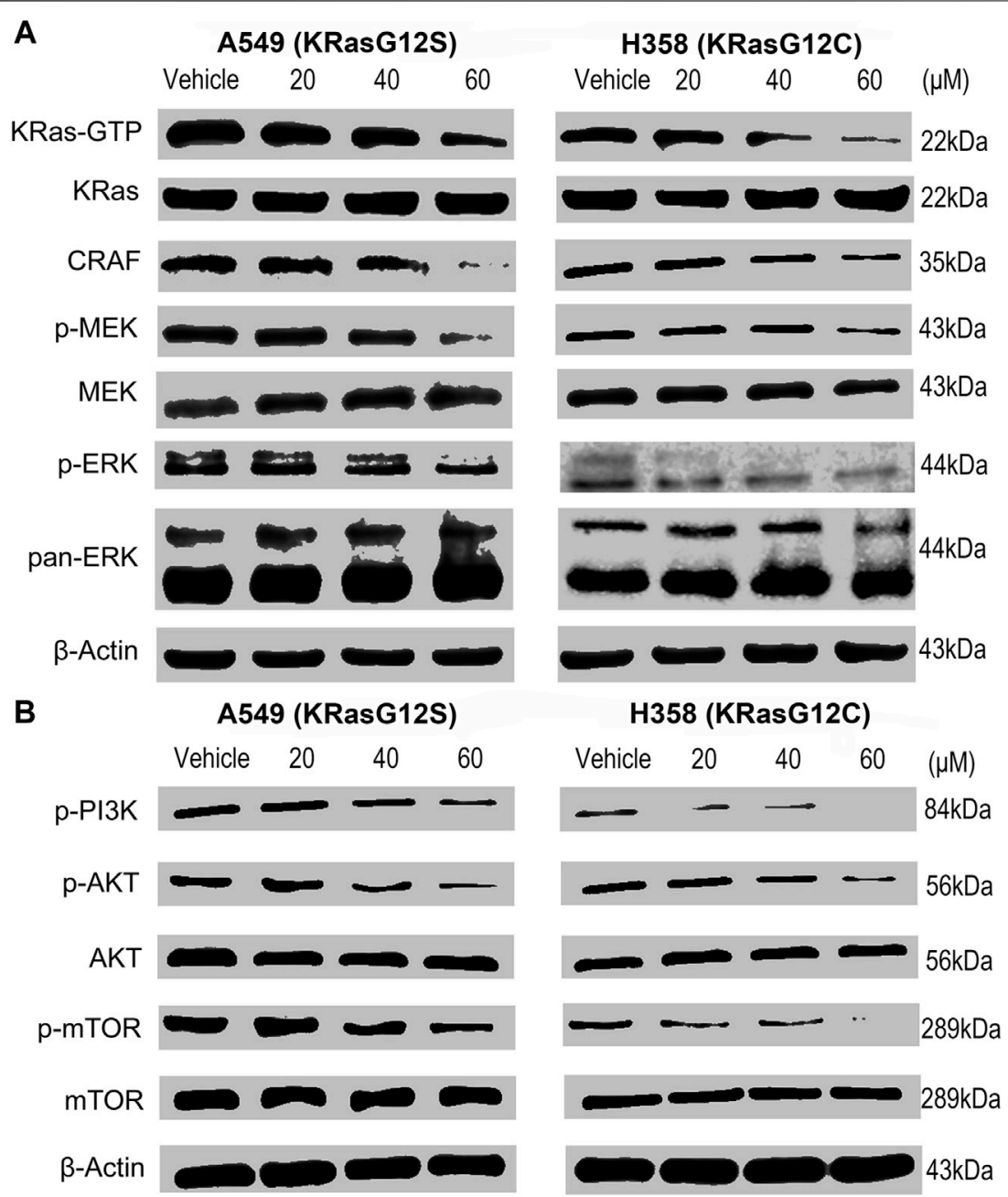

FIGURE 4 | NSC290956 inhibites KRas activity and downstream signaling events in KRas-driven nuytant NSCLC cells. (A) Pull-down of active GTP-bound KRas by Raf-RBD after exposure to NSC290956 showed a comparative decrease in the KRas-GTP and the RAF-MEK-ERK signaling. (B) KRas inactivation inhibited the PI3KAKT-mTOR signaling in both the cell lines. $\beta$-Actin was used as the internal control.

\section{NSC290956 Inhibited Cellular KRas Activity and Downstream Signaling Events}

In theory, NSC290956 binding to the "open conformation" might keep KRas at an inactive state and thereafter result in the cellular KRas-GTP attenuation. To verify this prediction, we evaluated the effect of NSC290956 blocking KRas activation on mutant A549 and H358 cell lines. These cells were serum-starved in the cultures and stimulated with EGF (that activates the MAPK cascade) before harvest. Thereafter, the KRas-GTP level was determined using an active KRas pull-down assay. As expected, a decrease of KRas-GTP was clearly detected in both the cell lines in a concentration-dependent manner, but no effect was observed on total KRas (Figure 4A).

We further found that both A549 and H358 cell lines showed the decreased CRAF abundance and the reduced MEK and ERK1/2 phosphorylations after NSC290956 treatment. By comparison, no obvious changes in the total MEK and pan-
ERK1/2 were detected in cells (Figure 4A). Simultaneously, the phosphorylations of $\mathrm{PI} 3 \mathrm{~K}, \mathrm{AKT}$, and $\mathrm{mTOR}$ were inhibited equally well (Figure 4B). In contrast, NSC290956 treatment did little to alter their total expressions when compared to control cells. The cell-based assays indicated that NSC290956 exerted a broad spectrum blockage of KRas downstream signaling events by direct blocking KRas activity (Supplementary Figure S3).

\section{NSC290956 Decreased the Viability, Cell Cycle, and Migration of Non-Small-Cell Lung Cancer Cells}

To assess the effect of NSC290956 on KRas-dependent cell survival, both A549 and H358 cells were respectively exposed to a range of NSC290956 concentrations, resulting in reduced viability (Figure 5A). We further tested a broader panel of 
A

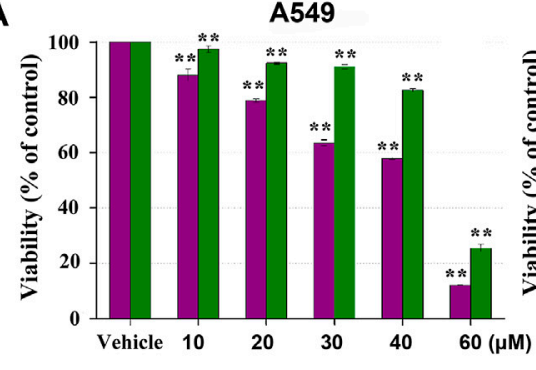

C

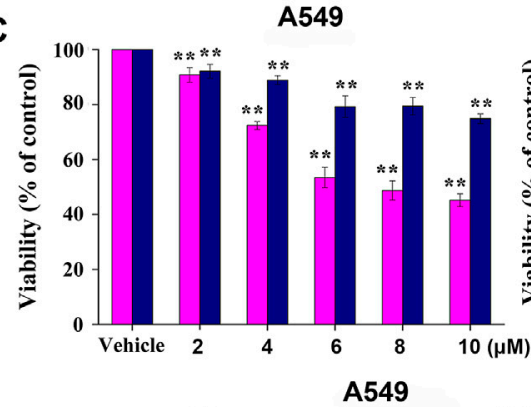

H358

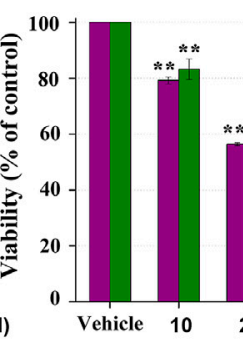

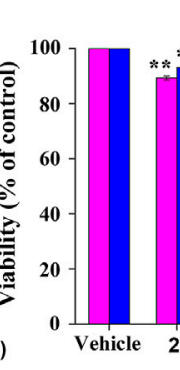

H358

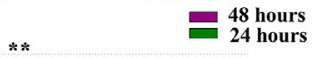

B
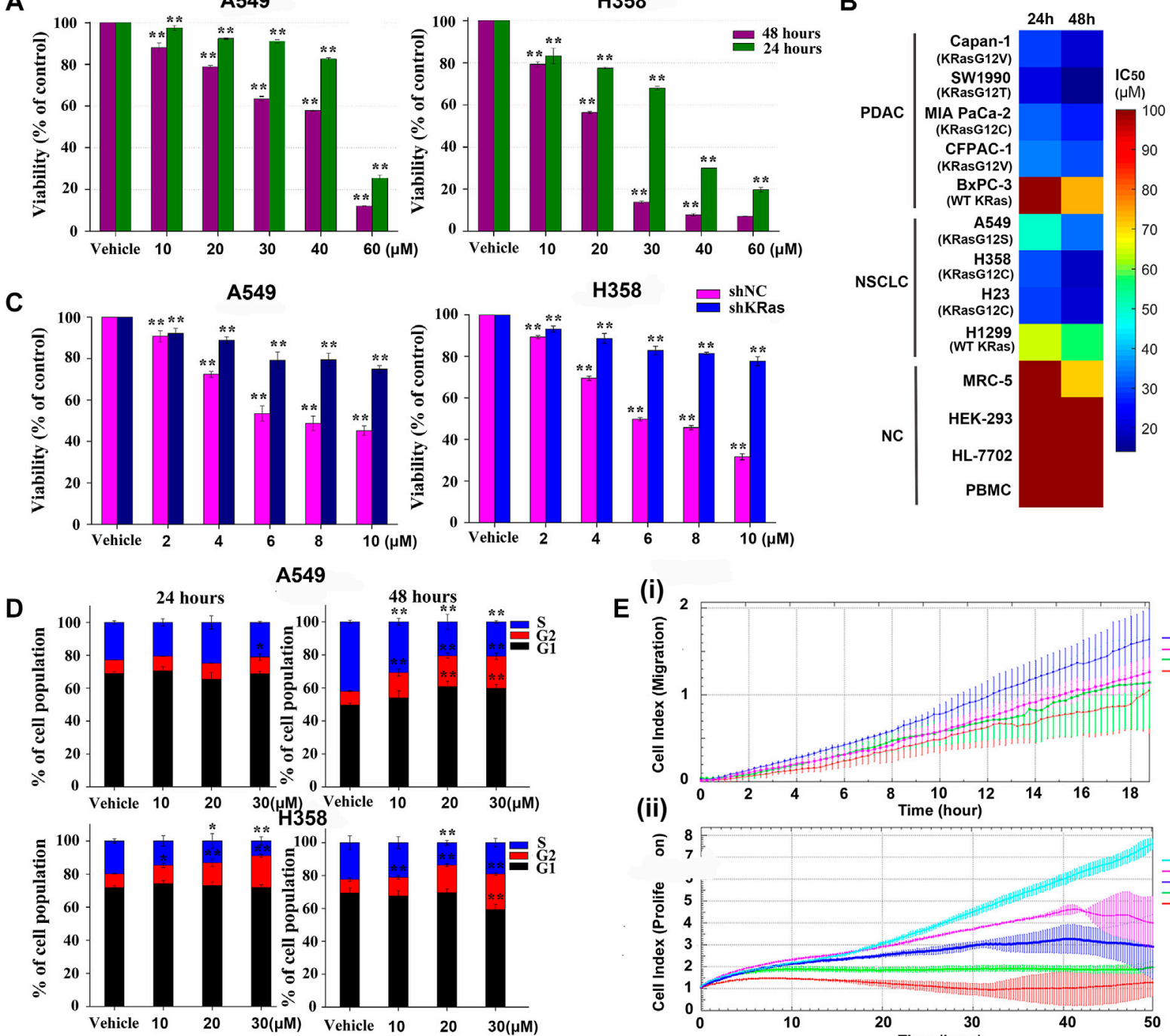

$E^{(i)}$

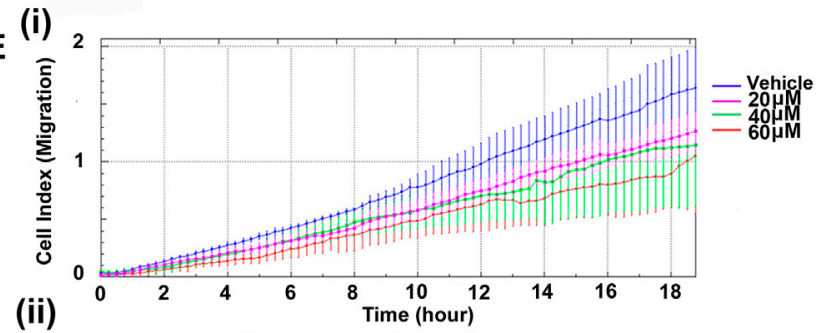

(ii)

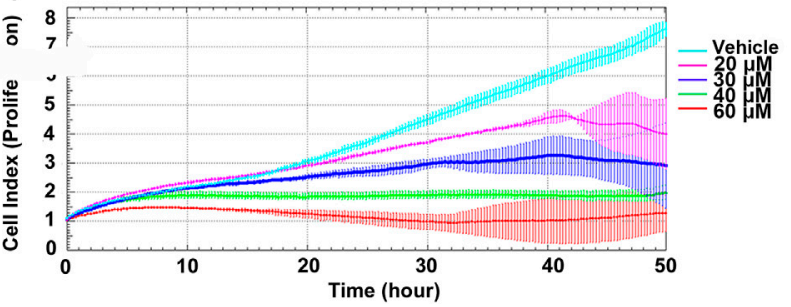

FIGURE 5 | NSC290956 decreases the cellular viability, cell cycle, and migration. (A) The survival effect of NSC290956 on representative A549 and H358 cell lines for 24 and 48 h, respectively. (B) A broader inhibitory effect of NSC290956 on various KRas-driven human pancreatic cancer (PDAC) cell lines (KRasG12V Capan-1, KRasG12T SW1990, KRasG12C MIA PaCa-2, KRasG12V CFPAC-1, and WT KRas BxPC-3), NSCLC cell lines (KRasG12S A549, KRasG12C H358, KRasG12C H23, and WT KRas H1299), and normal cells (NC) such as (MRC-5, HEK-293, HL-7702, and PBMC) was examined by exposure to NSC290956. (C) Effect of KRas deletion on NSC290956-induced growth inhibition in colony formation assay. Effect of shNC or shRNA plus drug treatment on colony formation was shown as the percentage of DMSO-treated control. (D) Effect of NSC290956 on cell cycle progression in the two cancer cell lines treated for 24 and 48 h, respectively. (E) Real-time cell index analysis of A549 cell migration over $18 \mathrm{~h}$ (i) and cell proliferation over $50 \mathrm{~h}$ (ii). The results were presented as the mean $\pm \mathrm{SD}$ of triplicate experiments. Significant differences from untreated control were indicated ${ }^{*} p<0.05$ and ${ }^{* *} p<0.01$.

KRas-driven mutant (KRasG12V CFPAC-1, KRasG12C MIA PaCa-2, KRasG12V Capan-1, KRasG12T SW1990 and KRasG12C H23) and wild-type KRas (BxPC-3 and H1299) cancer cells as well as human normal peripheral blood mononuclear cell (PBMC), embryonic kidney (HEK-293), lung fibroblast (MRC-5), and liver (HL-7702) cell lines. The dramatically reduced survival was observed in mutant KRasdriven cancer cells, while no significant growth inhibition was observed for the normal cells (Figure 5B). These observations raise the intriguing possibility that although all KRas mutations stabilize KRas-GTP levels, the different KRas oncogenic alleles may have distinct intrinsic hydrolysis rates of GTP.

To rule out the off-target effect, lentiviral short-hairpin RNA (shRNA) was introduced to suppress the expression of KRas in both cancer cell lines. The number of KRas in the shKRas cells was much less than that in the control or the negative control (shNC) cells (Supplementary Figures S4A,B). It's worth noting that both cell lines were sensitive to KRas knockdown (Supplementary Figure S4C). The effect of shRNA alone or plus drug treatment on colony formation was shown as the percentage of DMSO-treated control (Figure 5C). Knockdown 

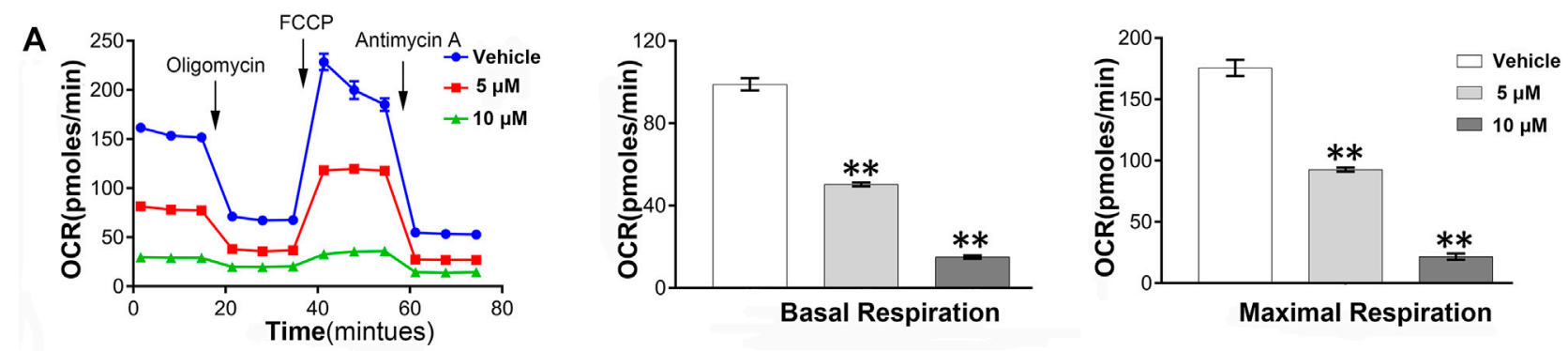

B
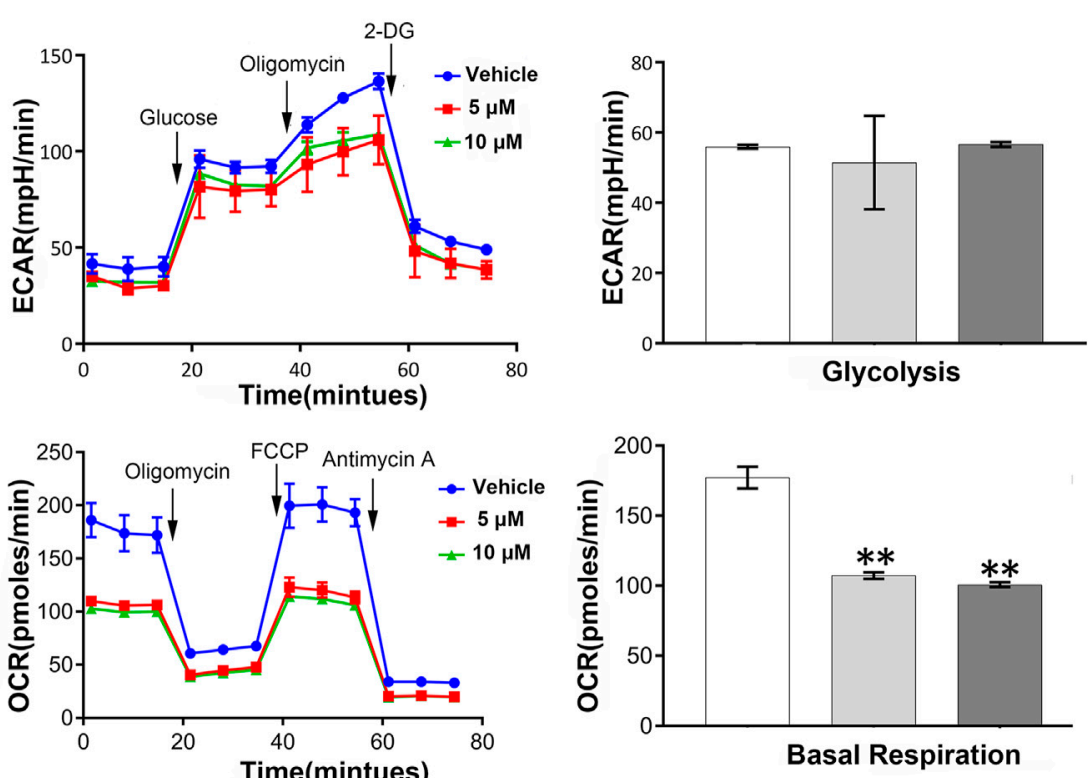

D
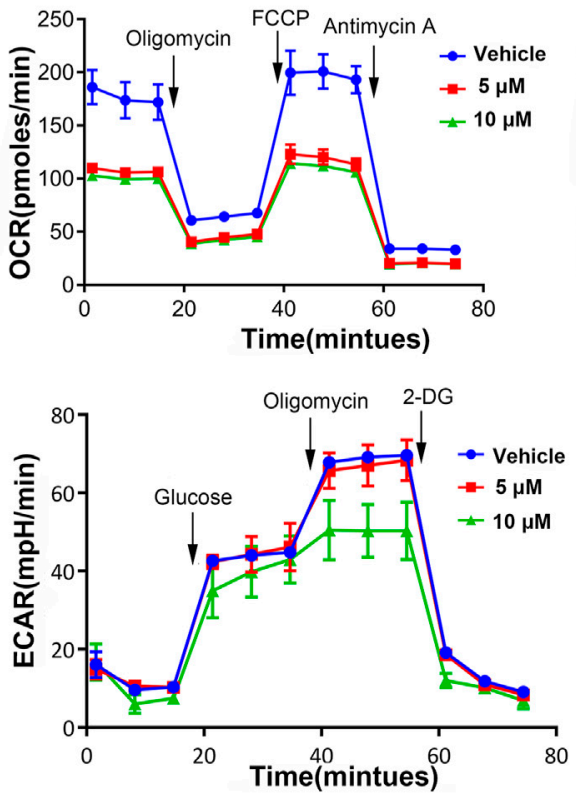

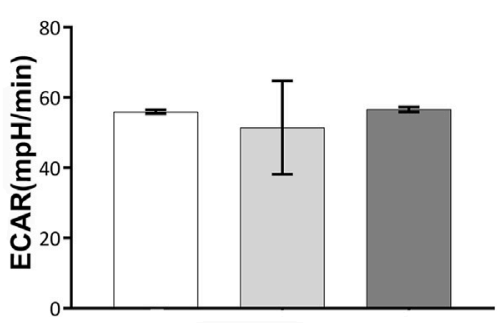

Glycolysis

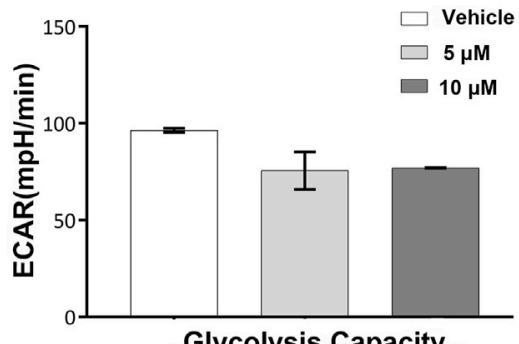

Glycolysis Capacity

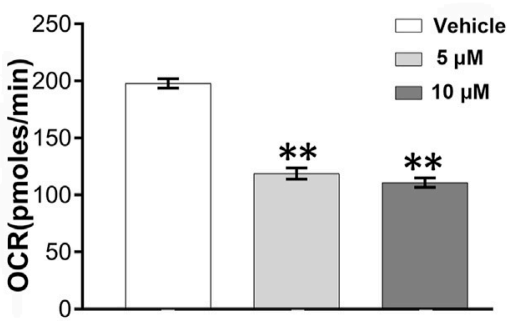

Maximal Respiration
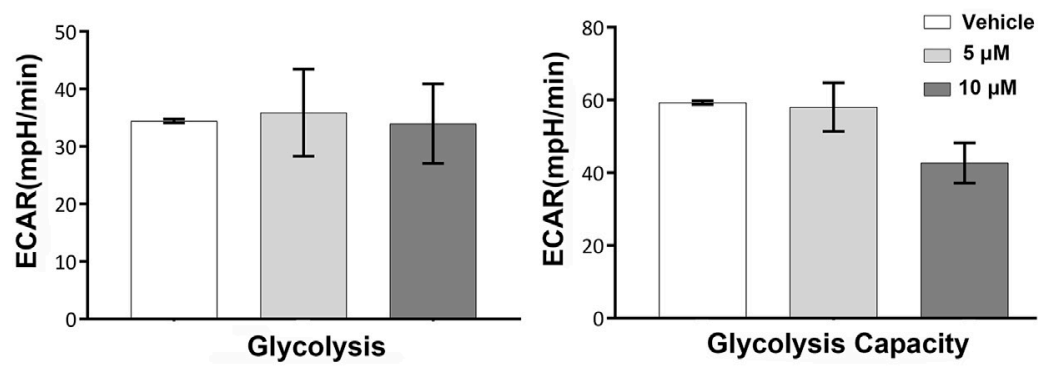

FIGURE 6 | Mitochondrial metabolism is required for KRas-mediated energy switching in both the cell lines. Representative basal OCR and ECAR values were measured in A549 cells (A,B) and H358 cells (C,D) in the presence of NSC290956. Seahorse assays were performed by using an XFp Extracellular Flux Analyzer. Cells were treated for $24 \mathrm{~h}$ then sequentially injected with $1 \mu \mathrm{M}$ oligomycin, $0.5 \mu \mathrm{M} \mathrm{FCCP}$, and $0.5 \mu \mathrm{M}$ anti-mycin A/0.5 $\mu \mathrm{M}$ rotenone for OCR test or with glucose, oligomycin, and 2-DG for ECAR test. Histogram data are the mean \pm SD of three replicates.

of KRas from these cells resulted in resistance to NSC290956, indicating a degree of KRas-dependent lethality. These findings support an on-target mechanism of action and a KRas-mutation specific way of function within the cells.

Simultaneously, the impact of NSC290956 on cell cycle related to cell growth was examined in A549 and H358 cell lines. A timeand concentration-dependent increase in the cell population at G2/M phase was observed intuitively (Figure 5D). CDK1 and Cyclin B1 expressions involving in $\mathrm{G} 2 / \mathrm{M}$ phase displayed the significantly decreased levels in the two cell lines (Supplementary Figure S5). It can be concluded that NSC290956 resulted in growth inhibition that elicited a prominent, prolonged accumulation of cells at G2/M phase in the cells. As an example, the real-time xCELLigence system analysis demonstrated that NSC290956-treated A549 cells showed the reduced migration in addition to the suppressed proliferation (Figure 5E). Of note, when the concentration was increased to $60 \mu \mathrm{M}$, cell growth was near complete abrogation in these cases. These results suggest that NSC290956 rather effectively 

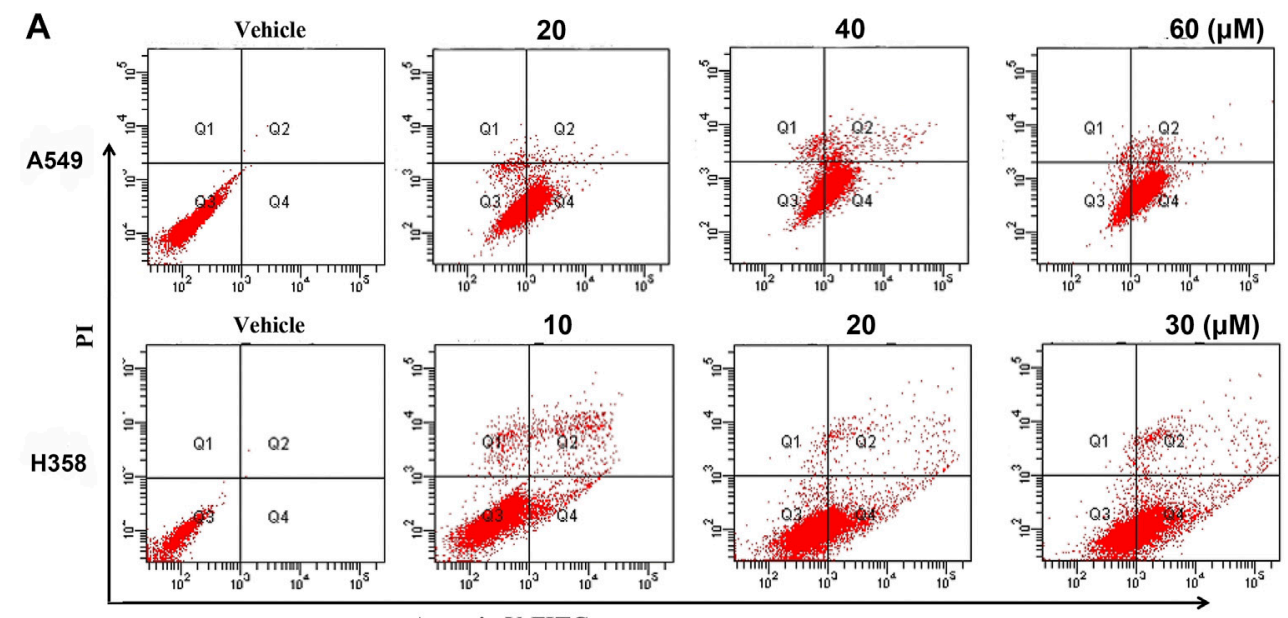

E
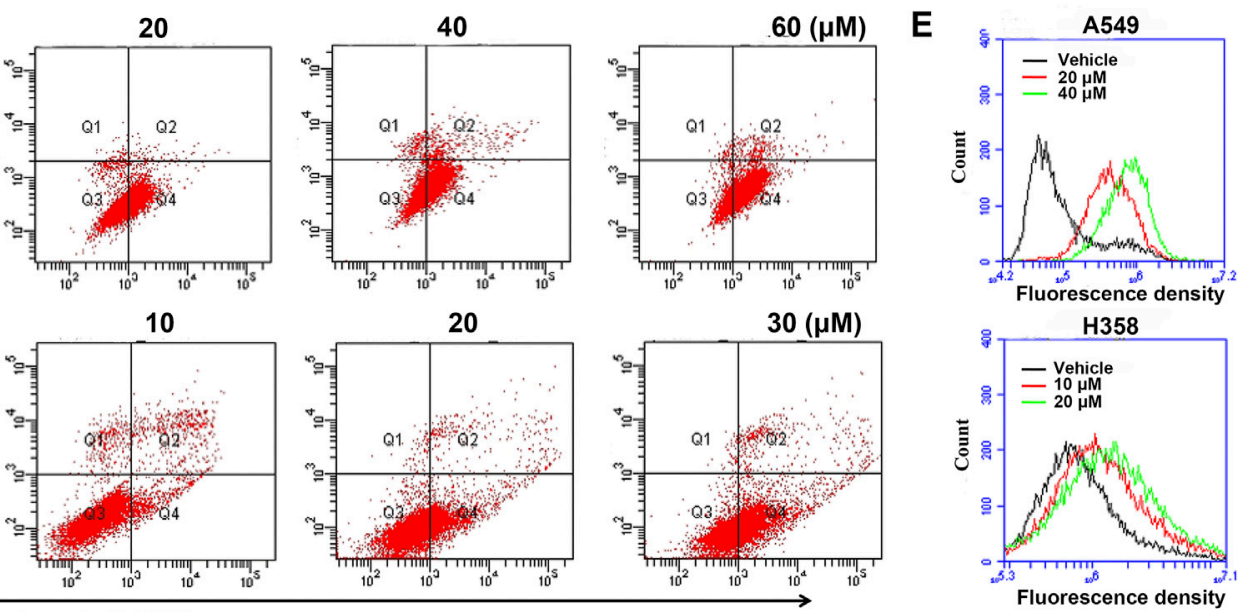

D

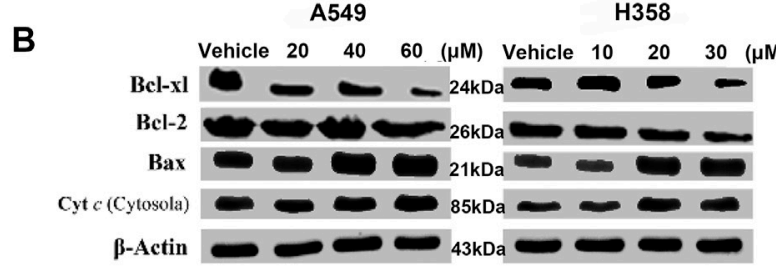

C
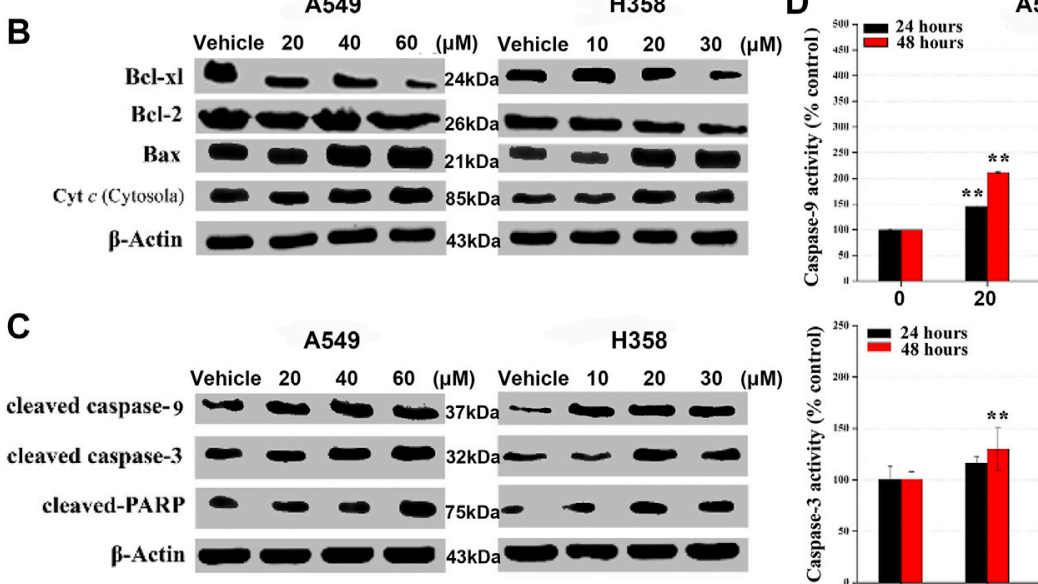

A549

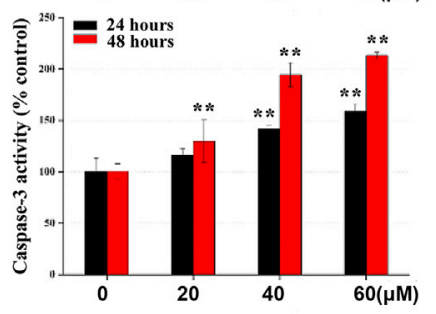

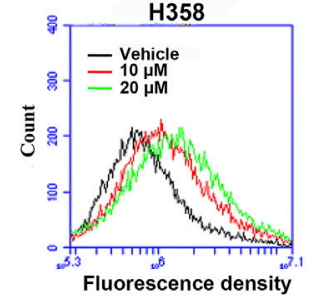

H358
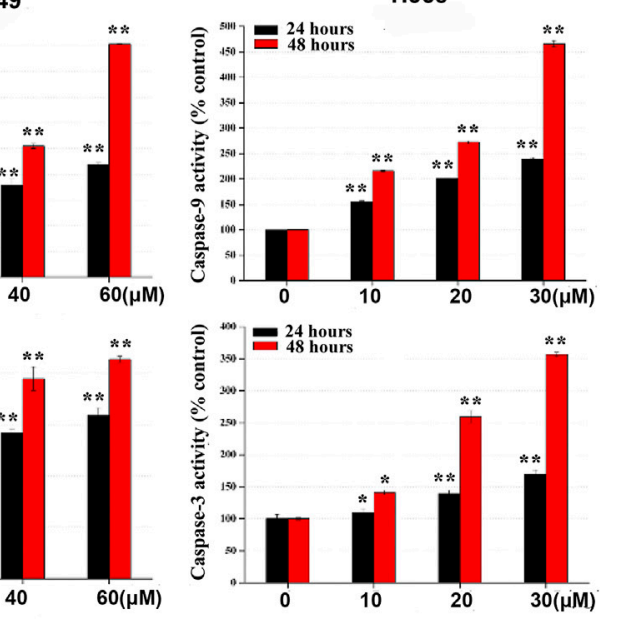

FIGURE 7 | NSC290956 regulates mitochondrial dysfunction for apoptosis in both A549 and H358 cells. (A) Apoptosis induced by the indicated dose of NSC290956 was measured by flow cytometry in both the cell lines, respectively. (B) Appearance of apoptosis markers in the cells was changed in NSC290956. Effects of NSC290956 on the activation of caspase-3 and caspase- 9 were assessed by WB analysis (C) and caspase activity assay (D), respectively. $\beta$-Actin was used as the internal control. Significant differences from untreated control were indicated ${ }^{*} p<0.05$ and ${ }^{\star \star} p<0.01$. (E) Flow cytometric detection of ROS generation is shown. $X$ axis-fluorescence density; $Y$ axis-cell amount.

suppresses the survival and proliferation of KRasG12C-driven H358 cells dependent phenotypes.

\section{NSC290956 Changed KRas-Dependent Metabolic Phenotype in Non-Small-Cell Lung Cancer Cells}

Metabolic regulation is essential for the maintenance of proliferation in tumor cell. We therefore questioned whether the anti-proliferation of NSC290956 is associated with metabolic phenotype switching between mitochondrial respiration and glycolysis in H358 and A549 cells. Applying Seahorse testing, the overall metabolic profiles, mitochondrial respiration and glycolysis, were examined by detecting the oxygen consumption rate (OCR) and the extracellular acidification rate (ECAR) in situ, respectively. In A549 cells, NSC290956 decreased the initial mitochondrial respiration induced by oncogenic KRas in a dose-dependent manner following the obviously reduced basal respiration and the maximal respiration capacity (Figure 6A). In contrast, glycolysis and glycolysis capacity were almost unchanged with the same concentrations of NSC290956 (Figure 6B). In addition, the metabolic regulation of NSC290956 was applicable to the other cell line with KRasG12C mutant H358 in a similar manner (Figures 6C,D). These results showed that NSC290956 produced a sustained decrease in mitochondrial metabolism. This provides a solid basis for the notion that mitochondrial metabolism is essential for KRasmediated tumor (Weinberg et al., 2010).

\section{NSC290956 Altered Mitochondrial Function for Apoptosis}

To validate if metabolic alterations may be responsible for cell proliferation inhibition, the events related to cell death were 


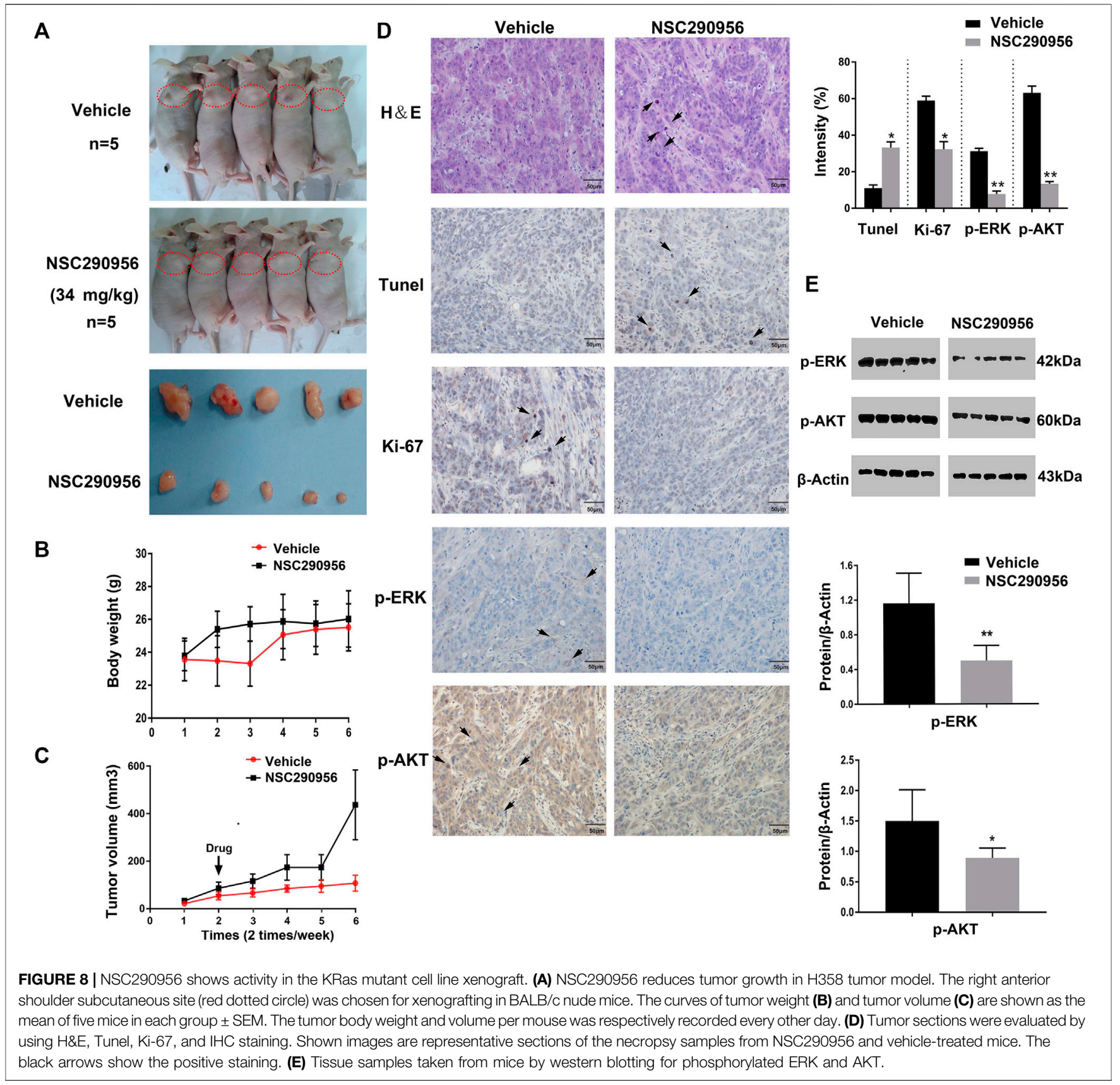

monitored as the readout of apoptosis. Flow cytometric data showed a dose-dependent apoptosis-induction effect of NSC290956 on both the cell lines, while the number of necrotic cells among all the cells remained small (Figure 7A; Supplementary Figure S6A). The apoptotic cell death was visually confirmed by cell imaging (Supplementary Figure S6B). WB analyses showed that the anti-apoptotic proteins $\mathrm{Bcl}-\mathrm{xl}$ and Bcl-2 were remarkably decreased, but the proapoptotic protein Bax was significantly increased (Supplementary Figure S7). In addition, NSC290956 obviously promoted the release of cytochrome $c$ into cytosol (Figure 7B). The released cytochrome $c$ accelerated caspase-9 activation, eventually triggering caspase-3 activation (Figures 7C,D). As an additional response mechanism, the mitochondrial membrane potential (MMP), an important indicator of mitochondrial health, was significantly decreased (Supplementary Figure S8) and the cellular reactive oxygen species (ROS) levels were markedly increased in a dosedependent manner upon NSC290956 treatment (Figure 7E). Consistent with the decreased mitochondrial metabolism, these results suggested that NSC290956 caused mitochondrial dysfunction. Thus, we propose that NSC290956-mediated cell death likely results from the mitochondria-dependent apoptosis. 
NSC290956 Reduced Tumor Growth in H358 Xenograft

We next addressed whether the target engagement achieved in the cell can translate to anti-tumor activity in the animal. The in vivo efficacy of NSC290956 was initially assessed in xenograft tumor model using H358 (KRasG12C) cells in nude mice. Based on previous renal capsule models of pancreatic cancer MIA $\mathrm{PaCa}-$ 2 (KRasG12C) cells in BALB/c mice (Guo et al., 2018), NSC290956 was administered by intraperitoneal injection with $34 \mathrm{mg} / \mathrm{kg}$ once per every day. The administration effectively reduced tumor growth for the duration of 2 weeks of treatment as compared to vehicle control (Figures 8A-C).

$\mathrm{H} \& \mathrm{E}$ (hematoxylin and eosin) and Ki-67 staining of tumor sections displayed a good cellularity and fewer Ki-67 positive cells compared to vehicle-treated cells (Figure 8D), revealing the efficacy after drug treatment in mice. Likewise, TUNEL staining in tumor sections showed a prominent increase of apoptotic cells in NSC290956-treated mice, which validated a pro-apoptotic contribution of NSC290956 to tumor growth inhibition. To confirm that the tumor growth inhibition is associated with inhibiting KRas signaling in vivo, these tumor samples were analyzed for markers of pharmacological KRas inhibition. Compared to vehicle-treated mice, the decreased phosphorylation levels of ERK and AKT were observed after NSC290956 administration. These results suggest that the in vivo efficacy of NSC290956 is related to the attenuations of MAPKERK and AKT-mTOR dual signaling (Figure 8E). Together, these results effectively demonstrated the treatment effectiveness of NSC290956 in the mutant KRas-driven NSCLC.

\section{DISCUSSION}

More than one-third of human tumors harbor active Ras mutations (Prior et al., 2012). Abnormal activation of Ras signaling in tumor indicates that it might be a key tumor target. These findings motivated extensive efforts to target the oncoproteins for tumor therapy. Regrettably, attempts to directly inhibit the oncogenic Ras came with little success (Ostrem and Shokat, 2016).

Extensive efforts have focused on targeting individual sites on the surface of Ras proteins by structure-based computational screening. Stockwell et al. applied a computational design approach to yield a multivalent pan-Ras inhibitor (compound 3144) that exhibited cellular lethality, partially dependent on Ras expression (Welsch et al., 2017). Liu et al. described a computational structure-based design and identification of ARS-1620, a covalent compound with high potency and selectivity for KRasG12C mutant cancers (Janes et al., 2018). Kataoka group reported the successful development of Kobe0065-family compounds by an in silico screening targeting a pock on M-Ras-GTP (Shima et al., 2013). These compounds may serve as scaffolds for the development of Ras inhibitors with higher potency and specificity. Here, we took advantage of the SPA screening approach to characterize and tackle the druggability of the "open conformation" with both affinity and specificity, which relies on the combination of in silico calculations, pharmacophore modeling, and molecular docking. The immediate outcome was the discovery of hit candidate NSC290956 for the first time. Besides NSC290956, other two compounds NSC48693 and NSC48160 had been identified to promote apoptosis in human pancreatic cancer cells harboring oncogenic KRas by the Figure 1 workflow. NSC48160 inhibited cell viability and colony formation in CFPAC-1 (KRasG12V) and BxPC-3 (wild-type KRas) cell lines (Li et al., 2013). NSC48693 had an ability to block the anchorage-independent growth of CFPAC-1, MIA PaCa-2, and BxPC-3 cancer cells in soft agar (Liu et al., 2012).

Given the predominant role of oncogenic KRas in tumor, therapeutic strategy either targeting the mutated KRas or inhibiting the excessive KRas signaling is valuable in the treatment of tumors. Targeting the "open conformation" therefore represents a breakthrough progress for developing a new KRas-targeted anti-tumor strategy. In theory, NSC290956 serves to bind to KRas and then to diminish the activated KRasGTP level. The current results revealed the KRas engagement of NSC290956 in intact cells by thermally stabilizing KRas (Figure 3D), further blocking the cellular KRas activity (Figure 4) and resulting in a degree of KRas-dependent lethality in NSCLC tumor cells (Figure 5A). Especially, it is worth noting that there are multiple relevant targets in cancer cells. It is impossible to exclude the possibility that NSC290956 blocks the KRas signaling events due to the unspecific interactions with other proteins. This will be explored in further work. NSC290956 at least manifested potent efficacy against the inhibition of KRas-mediated signaling, resulting in the dramatically reduced survival of the KRas-expressing human pancreatic cancer cell lines, including CFPAC-1 (KRasG12V), MIA PaCa-2 (KRasG12C), Capan-1 (KRasG12V), SW1990 (KRasG12T), and BxPC-3 (wild-type KRas) with $\mathrm{IC}_{50}$ ranging 19.7-74.2 $\mu \mathrm{M}$. However, little significant cytotoxicity was observed for human normal cell lines HEK293, HL7702, and PBMC with $\mathrm{IC}_{50}$ ranging 71.2-125.6 $\mu \mathrm{M}$. In this respect, targeting the "open conformation" may offer a much higher therapeutic index for selectively killing tumor cells while sparing healthy ones because of the discriminated inhibition of both cancerous and non-cancerous cells.

The interplay between metabolic remodeling and oncogenes has being appreciated in tumor. It is still elusive to understand the underlying mechanism for metabolic switching between cancer glycolysis and mitochondrial respiration, though progress has been made in this issue. Recently, Li and Wang developed an integrative network model to uncover the core role of oncogene Ras in the gene regulatory network and cancer-related metabolic pathway (Li and Wang, 2020). In silico calculations predicted that oncogenic KRas inactivation is a key factor for metabolic switching between mitochondrial and glycolytic metabolism in cancer. Herein we provide evidences that inactivating KRas with NSC290956 modifies metabolic features of KRas mutant A549 and $\mathrm{H} 358$ cells imposing repression of mitochondrial respiration without significant compensatory increase in glycolysis (Figure 6). The distinctive characteristics of the specific cell metabolism are in support of the view that disrupting the tumor metabolic phenotype can be exploited to preferentially 
kill the cancer cells. Obviously, the cytotoxic selectivity of NSC290956 between cancerous cells and normal cells as shown in Figure 5B can attribute to the characteristics of specific cell metabolism. This finding extends the current perspective that mitochondrial metabolism is essential for KRas-mediated tumor (Weinberg et al., 2010). These data indicate that the dysregulation of particular mitochondrial metabolic pathway may play a pivotal role in dictating which adaptive mechanism is therapeutic index in the treatment of a KRas-positive tumor. Further study is ongoing to enlarge this evidence on other KRas-mutated cancer cells with the final aim to evaluate whether NSC290956 can be a suitable therapeutic agent targeting metabolic vulnerability.

Following the in vitro observations in KRas-mutant NSCLC cells, the effect of NSC290956 was further evaluated in a subcutaneous tumor model using $\mathrm{H} 358$ tumor xenograft (Figure 8). In the case of $\mathrm{BALB} / \mathrm{c}$ nude mouse model, NSC290956 treatment led to the dramatically decreased tumor growth. The initial toxicity study in vivo showed no major toxicity using a chronic injection of NSC290956 into BALB/c nude mice, as it displayed that NSC290956 did not cause significant alteration in body weight loss. Guo et al. reported that Spiclomazine (i.e., NSC290956) more effectively reduced the tumor growth of MIA PaCa-2 in renal capsule xenografts than Gemcitabine-based treatment (Guo et al., 2018). Notably, in three of the five mice, the growth of tumors after Spiclomazine treatment was completely blocked. Especially, all of the Spiclomazine-treated mice did not show any body weight loss and other obvious signs of toxicity, and they were alive and healthy throughout the study during the treatment. In contrast, the Gemcitabine-treated mice showed a marked body weight loss and even death. These findings were in good agreement with the previous study that selective blocking of the activated KRas inhibited KRas transformation in animal models (Podsypanina et al., 2008). In NSC290956-treated mice, the ERK and AKT phosphoproteins in tumor sections were markedly decreased, which confirmed that the in vivo efficacy of NSC290956 is correlated with the impairment of KRas-mediated signaling. Together, the identification of NSC290956 determining the in vivo response to KRas is therefore timely for developing a better strategy to treat KRas-positive tumors.

\section{CONCLUSION}

There have been many attempts to simultaneously inhibit MAPK-ERK and AKT-mTOR dual pathways using a combination of inhibitors, which have generally failed to show an acceptable therapeutic index due to combined toxicity (Ostrem and Shokat, 2016). Our studies indicate that NSC290956 as a single agent shows a remarkable safety profile in spite of inhibiting dual signaling, which contributes to its unique mechanism of action. The current results demonstrate that drug discovery based on blocking Ras "open conformation" may be complementary to the therapeutic strategy of direct targeting Ras. This work bridges the gap between in silico virtual drug screening and proof-of-concept pre-clinical testing.

\section{METHODS}

\section{Materials and Reagents}

NSC290956 was dissolved in DMSO to make stock solution $(10 \mathrm{mg} / \mathrm{ml})$ and further diluted to appropriate concentrations with double distilled water containing $10 \%$ DMSO immediately before use. Dulbecco's phosphate buffered saline (PBS) was purchased from Sigma-Aldrich Inc. (St. Louis, MO). AnnexinV-propidium iodide (PI) apoptosis detection reagent was purchased from BD Bioscience (San Jose, CA). In addition, 5-(and 6)-chloromethyl-2' $2^{\prime}$-dichlorodihydrofluorescein diacetate (CM-H2DCFDA) and 4',6-Diamidino2-phenylindole (DAPI) were purchased from Invitrogen (Carlsbad, CA). Western blotting substrate was obtained from Thermo Scientific (Hudson, NH). Unless otherwise notified, all chemicals were obtained from Sigma-Aldrich. Polyvinylidenedifluoride (PVDF) membrane was purchased from EMD Millipore (Bedford, MA). Media (RPMI-1640 and DMEM) and fetal bovine serum (FBS) were purchased from Gibico (Grand Island, NY). Primary antibodies against human CRAF, MEK, phospho-MEK, ERK1/2, phospho-ERK1/2, AKT, phospho-AKT, phospho-PI-3K, phospho-mTOR, mTOR, Bcl-xl, Bcl-2, Bax, cytochrome c, cleaved caspase-9 and caspase-3, cleaved PARP, CDK1, Cyclin B1, Beclin1, KRas (C-terminal), and $\beta$-actin were all from Abcam Technology Inc. (Beverly, MA). The horseradish peroxidase-conjugated anti-rabbit or anti-mouse secondary antibodies were purchased from Santa Cruz Biotechnology Inc. (Santa Cruz, CA). Seahorse kits were purchased from Agilent.

\section{Cell Culture}

Human non-small cell lung carcinoma (H358 and A549) and pancreatic cancer (CFPAC-1, MIAPaCa-2, BxPC-3, SW1990, and Capan-1) cell lines were obtained from American Type Culture Collection (ATCC, Rockville, MD) and cultured in RPMI-1640 and DMEM or IMDM media supplemented with 10\% FBS and 100 units $/ \mathrm{ml}$ penicillin, $50 \mu \mathrm{g} / \mathrm{ml}$ streptomycin, and $100 \mu \mathrm{g} / \mathrm{ml}$ amphotericin (Invitrogen, Carlsbad, CA) at $37^{\circ} \mathrm{C}$ in $5 \% \mathrm{CO}_{2}$ incubator, respectively. Human normal embryonic kidney (HEK293), liver (HL-7702), and peripheral blood mononuclear cell (PBMC) cells were purchased from Chinese Academy of Science Type Culture Collection (Shanghai, China) and incubated in DMEM media containing $10 \%$ FBS. All the cells were maintained in $25 \mathrm{ml}$ flasks and kept in an atmosphere of $5 \%$ $\mathrm{CO}_{2}$ and $95 \%$ air under humidified conditions at $37^{\circ} \mathrm{C}$. The adherent cells other than PBMC were detached from the monolayer using $0.25 \%$ trypsin and $0.53 \mathrm{mM}$ EDTA for $5 \mathrm{~min}$ at $37^{\circ} \mathrm{C}$ when cells were grown to near confluence. All the cell lines were tested for mycoplasma contamination by using mycoplasma stain assay kit (Beyotime Institute of Biotechnology, China).

\section{SPecificity-Affinity Virtual Screening}

SPA, a two-dimension virtual screening strategy, is promising in searching for po-tential compounds due to its simultaneous reaching performance maximization on af-finity and specificity (Wang et al., 2007). HRasG60A-GppNp (PDB: 1XCM [Matsunaga et al., 2017], GppNp, Guanosine-5' -[( $\beta, \gamma)$-imido] triphosphate, a non-hydrolysable form of GTP) was used as the 
docking receptor in in silico calculations using Autodock3 package of programs (Morris et al., 1998). The cavity located between switch 1 region and residues Ile21, Gln25, Asp30, Tyr32, and Pro34 is defined as the binding pocket. The solvent parameters and partial charges were generated from the PRODRG 2 server (Schüttelkopf and van Aalten, 2004). GppNp and cofactor $\mathrm{Mg}^{2+}$ were retained for screening ligand. The water molecules interacting with $\mathrm{Mg}^{2+}$ were also retained. The other water, organic solvent, and lipid molecules were removed. Protons were automatically assigned in such a way that the protonation states of side chains and termini corresponded to $\mathrm{pH}$ 7.0. The de novo designs of compounds in the National Cancer Institute (NCI) database were carried out using the autogrow package according to scaffold of potential inhibitors (Zheng et al., 2013a; Zheng et al., 2013b). The ADME/ TOX filtering was carried out via the online FAF-Drugs2 tool (Lagorce et al., 2011). Pharmacophore models were constructed for compound using the LigandScout with default parameters (Wolber and Langer, 2005). The clustering analysis was carried out by AuPosSOM program (Bouvier et al., 2010).

In brief, SPA screening process for the "open conformation" was performed as displayed in the work flow (Figure 1). The affinity was calculated by measuring free energy difference before and after association. The specificity was calculated by measuring the gap $\delta \mathrm{E}$ (difference between the lowest energy and the average energy) and the spread or the variance of the energy spectrum $\Delta \mathrm{E}$ (Wang et al., 2007). We defined the intrinsic specificity ratio (ISR) $=\delta \mathrm{E} / \Delta \mathrm{E}$. Maximizing ISR is an optimization criterion for the binding specificity (Yan et al., 2013). Large ISR implies that the lowest energy (native binding) state is stable, significantly discriminated from the rest of the binding modes, and therefore specific. Since affinity may not always correlate with specificity, which is the ultimate goal for inhibitor design, ISR is used as an additional dimension for screening. Thus, compounds that had consensus free energy (high affinity) between -10 and $-8 \mathrm{kcal} / \mathrm{mol}$ as well as ISR value between 4 and 7.5 were chosen from the database. Finally, 26 hit candidates with high affinity and high specificity were selected and presented from NCI for next bioassay test. The most promising hit NSC290956 (also termed APY606 or Spiclomazine) was chosen for the final evaluation due to its minimum $\mathrm{IC}_{50}$ value.

\section{Confirmation of Protein by HPLC}

A $3.0 \mu \mathrm{l}$ aliquot of protein supernatant was injected using the Waters 1525 Binary HPLC Pump equipped with Waters 2998 Photodiode Array Detector. Mobile phase: $2 \mathrm{mM}$ ammonium formate aqueous solution containing $0.05 \%$ formic acid (buffer A) and methanol (buffer B). Flow rate: $500 \mu \mathrm{l} / \mathrm{min}$. Elution protocol: linear gradients $(0-1.0 \mathrm{~min}$ from $80 \% \mathrm{~A} / 20 \% \mathrm{~B}$ to $50 \% \mathrm{~A} / 50 \% \mathrm{~B}, 1-1.5 \mathrm{~min} 50 \% \mathrm{~A} / 50 \% \mathrm{~B}, 1.5-1.6 \mathrm{~min}$ from $50 \% \mathrm{~A} / 50 \% \mathrm{~B}$ to $80 \% \mathrm{~A} / 20 \% \mathrm{~B}, 1.6-3 \mathrm{~min}$ is $80 \% \mathrm{~A} / 20 \% \mathrm{~B}$ ).

\section{Western Blotting Analysis}

To clarify the underlying mechanisms of NSC290956 bioactivity at the molecular level, WB analysis was examined. H358 and A549 cell lines were seeded onto $100 \mathrm{~mm}$ plates. After being treated by NSC290956 as indicated concentrations, cells harvested were washed with cold PBS. After cellular proteins were extracted by using protein lysis kit (KeyGEN Biotech, Nanjing, China), protein concentrations were measured by Pierce BCA protein assay kit. Equal amount of total cellular protein $(30 \mu \mathrm{g})$ was separated on $10-12 \%$ SDS-PAGE gel and then transferred onto PVDF membrane. Thereafter, membrane was blocked in 5\% nonfat dry milk in TBST and probed with the relevant primary antibody (1:1,000 dilution) overnight at $4^{\circ} \mathrm{C}$. Visualization was performed using the appropriate horseradish peroxidase-conjugated secondary antibody (1:1,000 dilution). The specific proteins were scanned by using Chemiluminescence detector (DNR, KiryatAnavim, Israel). Protein level was normalized to the matching densitometric value of the internal control $\beta$-Actin.

\section{Cellular Thermal Shift Assay}

The ability of NSC290956 to interact with and stabilize KRas proteins in intact cells was analyzed essentially by CETSA. Briefly, $\mathrm{H} 358$ and A549 cells were grown in $100 \mathrm{~mm}$ plates to $70-80 \%$ confluency and then treated with cell media containing $0.1 \%$ DMSO and $12.5 \mu \mathrm{g} / \mathrm{ml}$ NSC290956 for $8 \mathrm{~h}$, respectively. After treatment, cells harvested were collected by centrifugation and subsequently resuspended in TBS buffer. Thereafter, these cells were suspended in lysis buffer and incubated on ice for $20 \mathrm{~min}$. Cells were then centrifuged at $14,000 \mathrm{rpm}$ for $15 \mathrm{~min}$ at $4^{\circ} \mathrm{C}$. The cell suspension was placed into microtubes and heated for $3 \mathrm{~min}$ to $44,46,48,50,52,54,56,58$, and $60^{\circ} \mathrm{C}$ followed by cooling for $3 \mathrm{~min}$ at room temperature. Precipitates were separated from the heated lysates by centrifugation at $14,000 \mathrm{rpm}$ for $20 \mathrm{~min}$ at $4^{\circ} \mathrm{C}$. The soluble protein super-natants were transferred to new microtubes and analyzed by SDS-PAGE followed by WB analysis using KRas (C-terminal) antibody at a concentration of 1:500.

\section{Cell Viability Assay}

The cell viability was assessed by Cell Counting Kit-8 (CCK-8) assay kit (Dojindo, Japan). Briefly, the cells seeded onto 96-well plates were treated by double distilled water containing DMSO as vehicle control groups or various concentrations of NSC290956 as experimental groups. Each concentration of a group of drugs was set five alternative holes. The cells were treated for 24 or $48 \mathrm{~h}$ and then the optical density (OD) at $450 \mathrm{~nm}$ was read with a M200 PRO NanoQuantautoreader (TECAN, Switzerland). The final concentration of DMSO in the culture media is $0.1 \%(\mathrm{v} / \mathrm{v})$, which does not significantly affect the cells.

\section{Flow Cytometric Detection of Apoptosis}

Based on the growth cycle of cancer cells, the cells were treated by NSC290956 for $24 \mathrm{~h}$ in order to discriminate the cell death as caused by either apoptosis induction or necrosis. Apoptotic H358 and A549 cells were quantified by Annexin V-fluorescein isothiocyanate (FITC)-propidium iodide (PI) apoptosis detection kit after cells were treated with double distilled water containing DMSO (final concentration of $0.1 \%$ ) as vehicle control groups or various concentrations of NSC290956 as experimental groups for $24 \mathrm{~h}$. Briefly, cells were trypsinized and washed twice with cold PBS, and then the cells were resuspended at a density of 
$1 \times 10^{6}$ cells $/ \mathrm{mL}$ in binding buffer. Thereafter, a quota of the cells were gently mixed and incubated in a $1.5 \mathrm{ml} \mathrm{EP}$ tube with $5 \mu \mathrm{l}$ annexin V-FITC and $5 \mu \mathrm{l}$ PI in the dark for $15 \mathrm{~min}$ at room temperature. A quota of binding buffer was then added to each tube and the cells were subjected to flow cytometric analysis (Accuri C6, Ann Arbor, MI). In total, 10,000 events were analyzed in each sample. Cells that stain positive for FITC annexin V and negative for PI are undergoing apoptosis (Q4 quadrant); cells that stain positive for both FITC annexin V and PI are either in the end stage of apoptosis (undergoing necrosis) (Q1 quadrant) or already dead (Q2 quadrant); and cells that stain negative for both FITC annexin V and PI are alive and not undergoing measurable apoptosis (Q3 quadrant).

\section{Apoptotic Nuclear Staining}

NSC290956-induced apoptotic nuclear condensation and morphological change were detected using DAPI staining. $\mathrm{H} 358$ and A549 cell lines were grown on glass-bottom plates to $50 \%$ confluence and then cultured in the medium in the presence of various concentrations of NSC290956 for $24 \mathrm{~h}$. Thereafter, the cells were fixed with $3.5 \%$ paraformaldehyde and then incubated in a fluid containing $2 \mathrm{mg} / \mathrm{ml}$ DAPI for $20 \mathrm{~min}$. The nuclear morphology of cells was observed by fluorescence microscopy (AMG EVOS, Life).

\section{Fluorescence Imaging of Cell Apoptosis}

Apoptosis of H358 and A549 cells seeded onto glass-bottomed plates was qualitatively measured using fluorescence microscopy (AMG EVOS, Life) at 488 and $543 \mathrm{~nm}$ to assess the FITC and PI signals. Cells that stain positive for FITC annexin $\mathrm{V}$ and negative for PI are undergoing apoptosis; cells that stain positive for both FITC annexin V and PI are either in the end stage of apoptosis (undergoing necrosis) or already dead; and cells that stain negative for both FITC annexin V and PI are alive and not undergoing measurable apoptosis.

\section{Measurement of Mitochondrial Membrane Potential $(\Delta \Psi \mathrm{m})$}

Disruption of $\Delta \Psi \mathrm{m}$ is a characteristic signature of apoptosis in mitochondrial-related pathway. $\Delta \Psi \mathrm{m}$ can be measured using fluorescent probe Rhodamine 123. Briefly, H358 and A549 cell lines were seeded into 6-well plates and treated with NSC290956 at different concentrations for $24 \mathrm{~h}$. Cells were washed with PBS and incubated in $1 \mathrm{mM}$ Rhodamine 123 solution for $20 \mathrm{~min}$. Thereafter, cells were resuspended in $500 \mu \mathrm{l}$ PBS buffer and subject to flow cytometer (Accuri C6, Ann Arbor, MI). In total, 10,000 events were analyzed in each sample. The decrease of fluorescence intensity indicates the loss of $\Delta \Psi \mathrm{m}$ in cancer cells.

\section{Measurement of Intracellular Reactive Oxygen Species Level}

Intracellular ROS level was measured by flow cytometric analysis as described previously. In brief, cells $\left(5 \times 10^{4}\right.$ cells/well $)$ were grown in 24 -well plates at $5 \% \mathrm{CO}_{2}$ and $37^{\circ} \mathrm{C}$ conditions. After treatment with vehicle or various concentrations NSC290956 for $24 \mathrm{~h}$, the cells were incubated with $5 \mu \mathrm{M}$ CM-H2DCFDA in PBS for $30 \mathrm{~min}$ and subjected to flow cytometry (Accuri C6, Ann Arbor, MI) to determine ROS levels at wavelength of 488 (excitation) and 530 (emission). In total, 10,000 events were analyzed in each sample.

\section{Cell Cycle Analysis}

To test the potent mechanism for NSC290956-induced cell growth inhibition, the effect of NSC290956 treatment on cell cycle distribution was explored by flow cytometry. Based on the duration of the cell cycle, it is feasible and reasonable to detect the cell cycle progression at the cell cycle checkpoint when cells are treated for $24 \mathrm{~h}$. In brief, $\mathrm{H} 358$ and A549 cell lines were seeded into six-well plates and treated with NSC290956 at the indicated concentrations for $24 \mathrm{~h}$, respectively. Cells were suspended and fixed in $70 \%(\mathrm{v} / \mathrm{v})$ ethanol at $4^{\circ} \mathrm{C}$ overnight. Thereafter, cells were washed with PBS, resuspended in $1 \mathrm{ml}$ of PBS containing $50 \mu \mathrm{g} / \mathrm{ml} \mathrm{PI} \mathrm{and} 1 \mathrm{mg} / \mathrm{ml} \mathrm{RNaseA}$ at room temperature in dark for $30 \mathrm{~min}$. In total, 10,000 events were analyzed immediately in each sample by flow cytometer (Accuri C6, Ann Arbor, MI). All experiments were repeated at least three times.

\section{Caspase Activity Assay}

Both $\mathrm{H} 358$ and A549 cells seeded in 6-well plates $\left(5 \% \mathrm{CO}_{2}\right.$, $37^{\circ} \mathrm{C}$ ) were pre-incubated overnight before NSC290956 was added to each well. After a period of exposure (24 or $48 \mathrm{~h}$ ) with various concentrations of NSC290956, cells harvested by trypsinization were washed by PBS and the activity of caspase- 3 and caspase- 9 was determined by caspase activity assay kit from Abcam Technology Inc. (Beverly, MA), respectively. The absorbance of enzymatically released pNA was measured at $490 \mathrm{~nm}$ on a M200 PRO NanoQuantautoreader (TECAN, Switzerland).

\section{Tumor Xenograft Models}

Femal athymic nude mice (6-8 weeks of age) were allowed to acclimate for 2 weeks in sterile micro isolator cages with constant temperature and humidity, with simultaneous free access to food and water $2 \times 10$ (Ford et al., 2005). H358 cells suspended in the fresh medium containing $10 \%$ Matrigel were injected subcutaneously per mouse. Tumors were allowed to grow to $\sim 50 \mathrm{~mm}^{3}$, and then mice were randomized into two groups (five per group). Mice were monitored daily and weighed twice weekly. Drug treatment started the day after inoculation. NSC290956 dissolved in DMSO was injected intraperitoneally every 2 days at $34 \mathrm{mg} / \mathrm{kg}$ for 2 weeks. DMSO with the same final concentration was injected intraperitoneally as vehicle-treated negative control. Mice were sacrificed at the end of the second week and the tumor from each mouse was excised. A portion of the tumors was fixed and processed for paraffin embedding and immunohistochemical (IHC) analysis. This animal protocol used in the current study was in accordance with the recommendation of the Chinese Academy of Sciences Ethics Committee and under the supervision of authorized investigators. 


\section{Statistical Analysis}

All datas were expressed as mean \pm SD of triplicate experiments, and reproducibility was confirmed in at least three separate experiments. Statistical analysis was performed by using GraphPad Prism Software version 7.04 (GraphPad, CA, United States). ${ }^{\star} p<$ 0.05 and ${ }^{\star *} p<0.01$ were considered statistically significant.

\section{DATA AVAILABILITY STATEMENT}

The original contributions presented in the study are included in the article/Supplementary Material, and further inquiries can be directed to the corresponding authors.

\section{ETHICS STATEMENT}

The animal study was reviewed and approved by the Animal Care and Use Committee of Wish Detection Technology Co., Ltd., Changchun, China (Approval Number: SYXK 2019-0007, Changchun, China).

\section{AUTHOR CONTRIBUTIONS}

Conceptualization, ZL, JW; methodology, software, validation, JZ, WZ, XY, XZ, and CL; formal analysis, JZ, XZ, ZL, and JW; investigation, JZ, ZL; writing original draft preparation, JZ, ZL, and JW; writing- review and editing, supervision, ZL and JW;

\section{REFERENCES}

Boriack-Sjodin, P. A., Margarit, S. M., Bar-Sagi, D., and Kuriyan, J. (1998). The Structural Basis of the Activation of Ras by Sos. Nature 394, 337-343. doi: $10.1038 / 28548$

Bouvier, G., Evrard-Todeschi, N., Girault, J. P., and Bertho, G. (2010). Automatic Clustering of Docking Poses in Virtual Screening Process Using SelfOrganizing Map. Bioinformatics 26, 53-60. doi:10.1093/bioinformatics/btp623

Burns, M. C., Sun, Q., Daniels, R. N., Camper, D., Kennedy, J. P., Phan, J., et al. (2014). Approach for Targeting Ras with Small Molecules that Activate SOSMediated Nucleotide Exchange. Proc. Natl. Acad. Sci. U.S.A. 111, 3401-3406. doi:10.1073/pnas.1315798111

Ford, B., Skowronek, K., Boykevisch, S., Bar-Sagi, D., and Nassar, N. (2005). Structure of the G60A Mutant of Ras: Implications for the Dominant Negative Effect. J. Biol. Chem. 280, 25697-25705. doi:10.1074/jbc.M502240200

Guo, X., Zhao, W., Liu, Z., and Wang, J. (2018). Spiclomazine Displays a Preferential Anti-Tumor Activity in Mutant KRas-Driven Pancreatic Cancer. Oncotarget 9, 6938-6951. doi:10.18632/oncotarget.24025

Hanahan, D., and Weinberg, R. A. (2011). Hallmarks of Cancer: The Next Generation. Cell 144, 646-674. doi:10.1016/j.cell.2011.02.013

Hobbs, G. A., Der, C. J., and Rossman, K. L. (2016). RAS Isoforms and Mutations in Cancer at a Glance. J. Cell. Sci. 129, 1287-1292. doi:10.1242/jcs.182873

Hwang, M. C., Sung, Y. J., and Hwang, Y. W. (1996). The Differential Effects of the Gly-60 to Ala Mutation on the Interaction of H-Ras p21 with Different Downstream Targets. J. Biol. Chem. 271, 8196-8202. doi:10.1074/jbc.271.14.8196

Janes, M. R., Zhang, J., Li, L. S., Hansen, R., Peters, U., Guo, X., et al. (2018). Targeting KRAS Mutant Cancers with a Covalent G12C-specific Inhibitor. Cell 172, 578-589.e17. doi:10.1016/j.cell.2018.01.006

Kano, Y., Gebregiworgis, T., Marshall, C. B., Radulovich, N., Poon, B. P. K., StGermain, J., et al. (2019). Tyrosyl Phosphorylation of KRAS Stalls GTPase Cycle project administration, JW and ZL; funding acquisition, ZL. All authors have read and agreed to the published version of the manuscript.

\section{FUNDING}

ZL thanks the supports from the Science Technology Development Plan of Jilin Province (20200301009RQ), the National Natural Science Foundation of China (81573448 and 21721003), the Ministry of Science and Technology of China (2016YFA0203200 and 2013YQ170585), the Scientific Instrument Developing Project of the Chinese Academy of Sciences (YJKYYQ20180038).

\section{ACKNOWLEDGMENTS}

Small molecule NSC290956 was partly supplied from NCI/DTP Open Chemical Repository (http://dtp.cancer.gov). The E.coli plasmids transformed were presented by Prof. A. Wittinghofer (Max Planck Institute of Molecular Physiology, Germany).

\section{SUPPLEMENTARY MATERIAL}

The Supplementary Material for this article can be found online at: https://www.frontiersin.org/articles/10.3389/fphar.2021.797821/ full\#supplementary-material

via Alteration of Switch I and II Conformation. Nat. Commun. 10, 224 doi:10.1038/s41467-018-08115-8

Lagorce, D., Maupetit, J., Baell, J., Sperandio, O., Tufféry, P., Miteva, M. A., et al. (2011). The FAF-Drugs2 Server: a Multistep Engine to Prepare Electronic Chemical Compound Collections. Bioinformatics 27, 2018-2020. doi:10.1093/ bioinformatics/btr333

Lenzen, C., Cool, R. H., Prinz, H., Kuhlmann, J., and Wittinghofer, A. (1998). Kinetic Analysis by Fluorescence of the Interaction between Ras and the Catalytic Domain of the Guanine Nucleotide Exchange Factor Cdc25Mm. Biochemistry 37, 7420-7430. doi:10.1021/bi972621j

Li, W., and Wang, J. (2020). Uncovering the Underlying Mechanisms of Cancer Metabolism through the Landscapes and Probability Flux Quantifications. iScience 23, 101002. doi:10.1016/j.isci.2020.101002

Li, D., Liu, Z., Zhao, W., Zheng, X., Wang, J., and Wang, E. (2013). A SmallMolecule Induces Apoptosis and Suppresses Metastasis in Pancreatic Cancer Cells. Eur. J. Pharm. Sci. 48, 658-667. doi:10.1016/j.ejps.2012. 12.023

Liu, Z., Li, D., Zhao, W., Zheng, X., Wang, J., and Wang, E. (2012). A Potent lead Induces Apoptosis in Pancreatic Cancer Cells. PLoS One 7, e37841. doi:10.1371/ journal.pone.0037841

Lu, J., Bera, A. K., Gondi, S., and Westover, K. D. (2018). KRAS Switch Mutants D33E and A59G Crystallize in the State 1 Conformation. Biochemistry 57, 324-333. doi:10.1021/acs.biochem.7b00974

Martinez Molina, D., Jafari, R., Ignatushchenko, M., Seki, T., Larsson, E. A., Dan, C., et al. (2013). Monitoring Drug Target Engagement in Cells and Tissues Using the Cellular thermal Shift Assay. Science 341, 84-87. doi:10.1126/ science. 1233606

Matsunaga, S., Hano, Y., Saito, Y., Fujimoto, K. J., Kumasaka, T., Matsumoto, S., et al. (2017). Structural Transition of Solvated H-Ras/GTP Revealed by Molecular Dynamics Simulation and Local Network Entropy. J. Mol. Graph Model. 77, 51-63. doi:10.1016/j.jmgm.2017.07.028 
Morris, G. M., Goodsell, D. S., Halliday, R. S., Huey, R., Hart, W. E., Belew, R. K., et al. (1998). Automated Docking Using a Lamarckian Genetic Algorithm and an Empirical Binding Free Energy Function. J. Comput. Chem. 19, 1639-1662. doi:10.1002/(SICI)1096-987X(19981115)19:14<1639:AID-JCC10>3.0.CO;2-B

Ostrem, J. M., and Shokat, K. M. (2016). Direct Small-Molecule Inhibitors of KRAS: From Structural Insights to Mechanism-Based Design. Nat. Rev. Drug Discov. 15, 771-785. doi:10.1038/nrd.2016.139

Pantsar, T. (2020). The Current Understanding of KRAS Protein Structure and Dynamics. Comput. Struct. Biotechnol. J. 18, 189-198. doi:10.1016/ j.csbj.2019.12.004

Parker, J. A., Volmar, A. Y., Pavlopoulos, S., and Mattos, C. (1993). K-ras Populates Conformational States Differently from its Isoform H-Ras and Oncogenic Mutant K-RasG12D. Structure 26, 810-e4. doi:10.1016/j.str.2018.03.018

Podsypanina, K., Politi, K., Beverly, L. J., and Varmus, H. E. (2008). Oncogene Cooperation in Tumor Maintenance and Tumor Recurrence in Mouse Mammary Tumors Induced by Myc and Mutant Kras. Proc. Natl. Acad. Sci. U.S.A. 105, 5242-5247. doi:10.1073/pnas.0801197105

Prior, I. A., Lewis, P. D., and Mattos, C. (2012). A Comprehensive Survey of Ras Mutations in Cancer. Cancer Res. 72, 2457-2467. doi:10.1158/0008-5472.CAN11-2612

Pylayeva-Gupta, Y., Grabocka, E., and Bar-Sagi, D. (2011). RAS Oncogenes: Weaving a Tumorigenic Web. Nat. Rev. Cancer 11, 761-774. doi:10.1038/ nrc3106

Schüttelkopf, A. W., and van Aalten, D. M. (2004). PRODRG: A Tool for HighThroughput Crystallography of Protein-Ligand Complexes. Acta Crystallogr. D Biol. Crystallogr. 60, 1355-1363. doi:10.1107/S0907444904011679

Shima, F., Yoshikawa, Y., Ye, M., Araki, M., Matsumoto, S., Liao, J., et al. (2013). In Silico discovery of Small-Molecule Ras Inhibitors that Display Antitumor Activity by Blocking the Ras-Effector Interaction. Proc. Natl. Acad. Sci. U.S.A. 110, 8182-8187. doi:10.1073/pnas.1217730110

Spoerner, M., Hozsa, C., Poetzl, J. A., Reiss, K., Ganser, P., Geyer, M., et al. (2010). Conformational States of Human Rat Sarcoma (Ras) Protein Complexed with its Natural Ligand GTP and Their Role for Effector Interaction and GTP Hydrolysis. J. Biol. Chem. 285, 39768-39778. doi:10.1074/jbc.M110.145235

Sun, Q., Burke, J. P., Phan, J., Burns, M. C., Olejniczak, E. T., Waterson, A. G., et al. (2012). Discovery of Small Molecules that Bind to K-Ras and Inhibit SosMediated Activation. Angew. Chem. Int. Ed. Engl. 51, 6140-6143. doi:10.1002/ anie. 201201358

Sung, Y. J., Hwang, M. C., and Hwang, Y. W. (1996). The Dominant Negative Effects of H-Ras Harboring a Gly to Ala Mutation at Position 60. J. Biol. Chem. 271, 30537-30543. doi:10.1074/jbc.271.48.30537
Wang, J., Zheng, X., Yang, Y., Drueckhammer, D., Yang, W., Verkhivker, G., et al (2007). Quantifying Intrinsic Specificity: A Potential Complement to Affinity in Drug Screening. Phys. Rev. Lett. 99, 198101-198104. doi:10.1103/ PhysRevLett.99.198101

Weinberg, F., Hamanaka, R., Wheaton, W. W., Weinberg, S., Joseph, J., Lopez, M., et al. (2010). Mitochondrial Metabolism and ROS Generation are Essential for Kras-Mediated Tumorigenicity. Proc. Natl. Acad. Sci. U.S.A. 107, 8788-8793. doi:10.1073/pnas.1003428107

Welsch, M. E., Kaplan, A., Chambers, J. M., Stokes, M. E., Bos, P. H., Zask, A., et al. (2017). Multivalent Small-Molecule Pan-RAS Inhibitors. Cell 168, 878. doi:10.1016/j.cell.2017.02.006

Wolber, G., and Langer, T. (2005). LigandScout: 3-D Pharmacophores Derived from Protein-Bound Ligands and Their Use as Virtual Screening Filters. J. Chem. Inf. Model. 45, 160-169. doi:10.1021/ci049885e

Yan, Z., Guo, L., Hu, L., and Wang, J. (2013). Specificity and Affinity Quantification of Protein-Protein Interactions. Bioinformatics 29, 1127-1133. doi:10.1093/ bioinformatics/btt121

Zheng, X., Liu, Z., Li, D., Wang, E., and Wang, J. (2013). Rational Drug Design: The Search for Ras Protein Hydrolysis Intermediate Conformation Inhibitors with Both Affinity and Specificity. Curr. Pharm. Des. 19, 2246-2258. doi:10.2174/ 1381612811319120012

Zheng, X., Gan, L., Wang, E., and Wang, J. (2013). Pocket-based Drug Design: Exploring Pocket Space. AAPS J. 15, 228-241. doi:10.1208/s12248-012-9426-6

Conflict of Interest: The authors declare that the research was conducted in the absence of any commercial or financial relationships that could be construed as a potential conflict of interest.

Publisher's Note: All claims expressed in this article are solely those of the authors and do not necessarily represent those of their affiliated organizations, or those of the publisher, the editors, and the reviewers. Any product that may be evaluated in this article, or claim that may be made by its manufacturer, is not guaranteed or endorsed by the publisher.

Copyright (c) 2022 Zhang, Liu, Zhao, Yin, Zheng, Liu, Wang and Wang. This is an open-access article distributed under the terms of the Creative Commons Attribution License (CC BY). The use, distribution or reproduction in other forums is permitted, provided the original author(s) and the copyright owner(s) are credited and that the original publication in this journal is cited, in accordance with accepted academic practice. No use, distribution or reproduction is permitted which does not comply with these terms. 